\title{
PROJECTIVE $n$-FOLDS OF LOG-GENERAL TYPE. I
}

\author{
M. BELTRAMETTI, A. BIANCOFIORE, AND A. J. SOMMESE
}

\begin{abstract}
Let $X$ be a normal $n$-dimensional Gorenstein irreducible projective subvariety of a complex projective space with codimension 3 singularities. This article introduces new projective invariants of $X$ and shows that they satisfy certain inequalities and relations making them useful tools in the projective classification of varieties. The main tools used are the recent precise adjunction theoretic results on classification of projective varieties.
\end{abstract}

\section{INTRODUCTION}

We are interested in the classification of projective varieties in terms of their projective invariants. In order to use the precise adjunction theoretic results that now exist without continually changing hypotheses, we assume that $X^{\wedge} \subset \mathbf{P}$ is a normal $n$-dimensional Gorenstein irreducible variety with codimension 3 singularities and $L^{\wedge}=\mathscr{O}_{\mathbf{P}}(1)_{\mid X^{\wedge}}$.

In [So7] it is shown that except for a small list of very well understood varieties there is a birational morphism $\pi: X^{\wedge} \rightarrow X$ with $X$ a normal Gorenstein $n$-fold and

(a) any positive dimensional fibre $F$ of $\pi$ is a $\mathbf{P}^{n-1}$ with $L_{\mathbf{P}^{n-1}}^{\wedge} \approx \mathscr{O}_{\mathbf{P}^{n-1}}(1)$ and $\pi(F)$ smooth,

(b) there exists a line bundle $L$ on $X$ such that

$$
K_{X^{\wedge}} \otimes L^{\wedge n-1} \approx \pi^{*}\left(K_{X} \otimes L^{n-1}\right)
$$

where $L$ is ample, $L=\left[\pi\left(A^{\wedge}\right)\right]$ for a general element $A^{\wedge} \in|L|$, $K_{X} \otimes L^{n-1}$ is ample and spanned by its global sections and in fact, very ample if $X^{\wedge}$ is smooth.

Such a pair $(X, L)$ is called a reduction of $\left(X^{\wedge}, L^{\wedge}\right)$.

Except for a second small list of very well understood pairs $\left(X^{\wedge}, L^{\wedge}\right)$, $K_{X} \otimes L^{n-2}$ is semi-ample and big. If $n=3$, this means that $X \backslash A$ for a general $A \in|L|$ or equivalently $X^{\wedge} \backslash A^{\wedge}$ for a general $A^{\wedge} \in\left|L^{\wedge}\right|$ is of log-general type. The main problem of the projective classification lies with the pairs $(X, L)$.

Received by the editors July 1, 1987 and, in revised form, November 1, 1987 and March 10, 1988.

1980 Mathematics Subject Classification (1985 Revision). Primary 14N99, 14J99; Secondary $14 \mathrm{C} 20$. 
Smooth surfaces $S$ given by transverse intersection of $n-2$ general members of $|L|$ are minimal models of general type.

We let $\mathscr{K}_{X}=K_{X} \otimes L^{n-2}$ and define the projective invariants

$$
d_{j}=\mathscr{K}_{X}^{j} \cdot L^{n-j}
$$

for $j=0, \ldots, n$. For a smooth $S$ as above it is easy to see that $d_{2}=K_{S} \cdot K_{S}$, $d_{1}=K_{S} \cdot L_{S}$, and that a smooth curve $C$ obtained as the intersection of $n-1$ general members of $|L|$ has genus $g(L)$ where $2 g(L)-2=d+d_{1}$, $d=d_{0}$. Note $g(L)=g\left(L^{\wedge}\right)$. All these $d_{j}$ are positive and are linked by Hodge inequalities

$$
d_{j+1} d_{j-1} \leq d_{j}^{2} \text { for } j=1, \ldots, n .
$$

One main result is that for such pairs $(X, L), K_{X}+n \mathscr{K}_{X}$ is effective except if $n=3,4$, or $6, \mathscr{K}_{X}$ is spanned by global sections, and $\Gamma\left(\mathscr{K}_{X}\right)$ gives a birational map $\phi: X \rightarrow \mathbf{P}^{n}$. For these special classes we compute all possible invariants $d_{j}$ 's. E.g. if $n=3$ then $d_{3}=1, d_{2}=5, d_{1} \leq 25, d \leq 125$ and a smooth $S \in|L|$ has the same Hodge $(p, q)$ numbers as a smooth degree 5 surface in $\mathbf{P}^{3}$. In this case $d_{1}$ and $d$ can vary quite a lot. We give a few examples, one with $d=13$ and $d_{1}=9$. The only cases when $d=125, d_{1}=25$ are the degree 5 surfaces in $\mathbf{P}^{3}$. The fact that, except for the above special varieties, $K_{X}+n \mathscr{K}_{X}$ is effective gives us a new and very useful inequality

$$
(n+1) d_{j} \geq(n-2) d_{j-1}, \quad j \geq 1 .
$$

Note also that $d_{j}$ and $d_{j+1}$ have the same parity if $j$ is even. These inequalities imply for example that

$$
d^{\wedge} \leq d \leq(n+1) \frac{2 g(L)-2}{2 n-1}
$$

where $d^{\wedge}=\left(L^{\wedge}\right)^{n}$ and, e.g. for $n=3$,

$$
K_{S} \cdot K_{S} \geq(g(L)-1) / 10 .
$$

In terms of the notion of spectral value $\sigma(X, L)$ of a pair $(X, L)$ introduced in [So7] (see §1), these results show that if $\sigma(X, L)>3$ then the effectivity of $K_{X}+n \mathscr{K}_{X}$ gives the inequality $\sigma(X, L) \geq(4 n+1) /(n+1)$. Previously pairs with $\sigma(X, L) \leq 3$ have been classified.

In the particular case when $n=3$ and $X$ is smooth, we use the effectivity of $K_{X}+n \mathscr{K}_{X}$ and the semipositivity of appropriate combinations of Chern classes of the first jet bundle $J_{1}(X, L)$ of $L$ to obtain other inequalities (see also [So4, Li-So]).

Finally we include a list of invariants for threefolds in $\mathrm{P}^{5}$ with $g(L) \leq 14$. To get such a list we use a Pascal program using all the above inequalities, plus other tricks discussed in the paper, running on a VAX 750 of the College of Science of the University of Notre Dame. 
In $\S 0$ we summarize background material that is not so well known in the form that we use it. We also explain the notion of reduction and give references for the adjunction theoretic results we use.

In $\S 1$ we give the basic theory of the $d_{j}$ 's and in $\S 2$ we prove the effectivity result for $K_{X}+n \mathscr{K}_{X}$ discussed above.

In $\S 3$ we give numerical classification results in the three dimensional case. The search for results that allows us to effectively enumerate the numerical invariants of projective varieties has been a major, if not the main, reason for the development of adjunction theory (see also [R]).

We would like to thank the referee for his suggested improvements of Theorem (2.2); we originally only gave the proof for dimension three.

We would like to thank the University of Notre Dame and the Universities of Genova and L'Aquila for their help in making our collaboration possible. We are grateful to Kenneth Grant for his help in running our jobs on the VAX's of the College of Science of the University of Notre Dame. The third author would like to thank the National Science Foundation (DMS 8420 315) for its financial support.

\section{NOTATION AND BACKGROUND MATERIAL}

We work over the complex number $\mathbf{C}$. By variety we mean an irreducible and reduced projective scheme $X$ of dimension $n$. We denote its structure sheaf by $\mathscr{O}_{X}$. For any coherent sheaf $\mathscr{F}$ on $X, h^{i}(\mathscr{F})$ denotes the complex dimension of $H^{i}(X, \mathscr{F})$.

If $X$ is normal, the dualizing sheaf $K_{X}$ is defined to be $j_{*} K_{\operatorname{Reg}(X)}$ where $j: \operatorname{Reg}(X) \rightarrow X$ is the inclusion of the smooth points of $X$, and $K_{\operatorname{Reg}(X)}$ is the canonical sheaf of holomorphic $n$-forms. Note that $K_{X}$ is a line bundle if $X$ is Gorenstein. We denote by $\operatorname{Irr}(X)$ the irrational locus of $X$.

Let $\mathscr{L}$ be a line bundle on a normal variety $X . \mathscr{L}$ is said to be numerically effective (nef, for short) if $\mathscr{L} \cdot C \geq 0$ for all effective curves $C$ on $X$, and in this case $\mathscr{L}$ is said to be big if $c_{1}(\mathscr{L})^{n}>0$ where $c_{1}(\mathscr{L})$ is the first Chern class of $\mathscr{L}$. We say that $\mathscr{L}$ is semi-ample if $\mathscr{L}^{t}$ is spanned by global sections for some positive integer $t$. We shall denote by $|\mathscr{L}|$ the complete linear system associated to $\mathscr{L}$ and by $\Gamma(\mathscr{L})$ the space of global sections.

(0.1) If $\mathscr{L}$ is a nef and big line bundle on a normal variety $X$, then a convenient form (see [So7, (0.2.1)]) of the Kawamata-Viehweg vanishing theorem is

$$
h^{i}\left(K_{X} \otimes \mathscr{L}\right)=0 \text { for } i>\max (0, \operatorname{dim} \operatorname{Irr}(X)) .
$$

(0.2) We fix some more notation:

$\sim$ (resp. $\approx$ ), the numerical (resp. linear) equivalence of line bundles;

$\chi(\mathscr{L})=\sum(-1)^{i} h^{i}(\mathscr{L})$, the Euler characteristic of a line bundle $\mathscr{L}$;

$e(X)=c_{n}(X)$, the topological Euler characteristic of $X$, for $X$ smooth, where $c_{n}(X)$ is the $n$th Chern class of the tangent bundle $\mathscr{T}_{X}$ and hence of $X$. 
Abuses. Line bundles and divisors are used with little (or no) distinction. Hence we shall freely switch from the multiplicative to the additive notation and vice versa. Sometimes the symbol "." of intersection of cycles is understood.

(0.3) For a line bundle $\mathscr{L}$ on a normal variety $X$, the sectional genus $g(\mathscr{L})$ of $(X, \mathscr{L})$ is defined by

$$
2 g(\mathscr{L})-2=\left(K_{X}+(n-1) \mathscr{L}\right) \cdot \mathscr{L}^{n-1} .
$$

(0.4) Lemma. Let $\mathscr{L}$ be a line bundle on a normal variety $X$. Then the sectional genus $g(\mathscr{L})$ is an integer number.

Proof. Choose an integer $t, t$ even and large, an ample line bundle $M$ on $X$ such that $\mathscr{L}+t M$ is very ample. Then $g(\mathscr{L}+t M)$ is an integer and the genus formula gives

$$
\begin{aligned}
2 g(\mathscr{L}+t M) & =\left(K_{X}+(n-1)(\mathscr{L}+t M)\right) \cdot(\mathscr{L}+t M)^{n-1} \\
& =\left(K_{X}+(n-1) \mathscr{L}\right) \cdot \mathscr{L}^{n-1} \bmod (2) \\
& =2 g(\mathscr{L})-2 \bmod (2) .
\end{aligned}
$$

Therefore $g(\mathscr{L})$ is an integer.

(0.5) Spectral value. Let $\mathscr{L}$ be an ample line bundle on a normal variety $X$ of dimension $n$. Following [So7] we define the spectral value of the polarized pair $(X, \mathscr{L})$ as the smallest real number $\tau$ such that given any fraction $p / q>\tau$

$$
h^{0}\left(m\left(K_{X}+(n+1-p / q) \mathscr{L}\right)\right)=0
$$

for all integers $m>0$ with $q \mid m$.

(0.6) Let $X^{\wedge}$ be a normal Gorenstein variety of dimension $n \geq 2$ and let $L^{\wedge}$ be a very ample line bundle on $X^{\wedge}$. If $n \geq 3$, let us assume that $\operatorname{cod} \operatorname{Sing}\left(X^{\wedge}\right) \geq 3$ and $\operatorname{dim} \operatorname{Irr}\left(X^{\wedge}\right)=0$. By a Bertini type theorem (see [So7, §0]) the transverse intersection of $n-2$ (resp. $n-1)$ general elements of $\left|L^{\wedge}\right|$ is a smooth surface $S^{\wedge}$ (resp. a smooth curve $C^{\wedge}$ ).

(0.7) Reductions [So7, (0.5)]. Let $\left(X^{\wedge}, L^{\wedge}\right)$ be as in $(0.6)$. We say that a pair $(X, L)$ is a reduction of $\left(X^{\wedge}, L^{\wedge}\right)$ if $L$ is ample and

(0.7.1) there exists a morphism $\pi: X^{\wedge} \rightarrow X$ expressing $X^{\wedge}$ as $X$ with a finite set $F$ blown up, $F$ contained in $\operatorname{Reg}(X)$;

(0.7.2) $L^{\wedge} \approx \pi^{*} L-\left[\pi^{-1}(F)\right]$, or, equivalently,

$$
K_{X^{\wedge}}+(n-1) L^{\wedge} \approx \pi^{*}\left(K_{X}+(n-1) L\right)
$$

Note that there is a one-to-one correspondence between elements $A^{\wedge} \in\left|L^{\wedge}\right|$ which are smooth in a neighbourhood of $\pi^{-1}(F)$ and elements $A \in|L|$ such that $F \subset \operatorname{Reg}(A)$. The correspondence is gotten by sending such an $A$ to its proper transform $A^{\wedge}$.

(0.8) Semi-ampleness of $K_{X}+(n-2) L$. Write $\mathscr{K}_{X}=K_{X}+(n-2) L$ and let $(X, L)$ be a reduction of $\left(X^{\wedge}, L^{\wedge}\right)$ as in (0.7), with $n \geq 3$. Then we have (compare with (1.2.2)) the following proposition. 
(0.8.1) Proposition. Let $\mathscr{K}_{X}$ be nef and big. Then $\mathscr{K}_{X}^{t}$ is spanned by global sections for $t \geq 5$.

Proof. First note that $\Gamma\left(\mathscr{K}_{X}^{t}\right)$ spans $\mathscr{K}_{X}^{t}$ on $\operatorname{Irr}(X)$ for all positive integers $t$ (see [So6]). Thus let $x \in X \backslash \operatorname{Irr}(X)$. Choose a general $A \in|L|$ with $x \in A$. Note that $A$ is normal and Gorenstein by Bertini (see [So7, $\S 0]$ ). Consider the exact sequence

$$
0 \rightarrow K_{X} \otimes L^{n-3} \otimes \mathscr{K}_{X}^{t} \rightarrow \mathscr{K}_{X}^{t+1} \rightarrow \mathscr{K}_{A}^{t+1} \rightarrow 0 .
$$

Now $h^{1}\left(K_{X} \otimes L^{n-3} \otimes \mathscr{K}_{X}^{t}\right)=0$ by the Kodaira vanishing theorem, hence

$$
\Gamma\left(\mathscr{K}_{X}^{t+1}\right) \rightarrow \Gamma\left(\mathscr{K}_{A}^{t+1}\right) \rightarrow 0
$$

is onto for $t \geq 1$ (note $\mathscr{K}_{A}=\mathscr{K}_{X \mid A}$ ). Therefore sections lift and by induction it suffices to prove this for $n=3$. Thus we are reduced to proving the following lemma.

(0.8.2) Lemma. Let $S$ be a normal Gorenstein surface with only rational singularities. If $K_{S}$ is nef and big, then $K_{S}^{t}$ is spanned by global sections for $t \geq 5$.

Proof. This is due to Bombieri. Let $p: S^{\prime} \rightarrow S$ be the minimal desingularization of $S$. Note $K_{S^{\prime}}=p^{*} K_{S}$, so that $K_{S^{\prime}}$ is nef and big. Then the result follows from [Bo].

(0.8.3) Corollary. There exist a normal projective variety $Y$, an ample line bundle $\mathscr{M}$ on $Y$ and a proper modification $r: X \rightarrow Y$ such that $\mathscr{K}_{X}=r^{*} \mathscr{M}$.

Proof. Let sor: $X \rightarrow Y \rightarrow \mathbf{P}^{N}$ be the Remmert-Stein factorization associated to $\Gamma\left(\mathscr{K}_{X}^{5}\right)$. Since $\mathscr{K}_{X}^{5}=r^{*} \mathscr{M}_{5}$ for some ample line bundle $\mathscr{M}_{5}$ on $Y$ and since the Remmert-Stein factorization is independent of $t$ if $\mathscr{K}_{X}^{t}$ is spanned one has also $\mathscr{K}_{X}^{6}=r^{*} \mathscr{M}_{6}$ for some ample line bundle $\mathscr{M}_{6}$ on $Y$. Then $\mathscr{K}_{X}=r^{*}\left(\mathscr{M}_{6} \otimes \mathscr{M}_{5}^{-1}\right)$.

(0.9) Theorem (Sommese). Let $\left(X^{\wedge}, L^{\wedge}\right)$ and $S^{\wedge}$ be as in (0.6) and let us assume that $K_{X^{\wedge}}+(n-1) L^{\wedge}$ is nef and big. Then

(0.9.1) there exists a reduction $\pi,(X, L)$ of $\left(X^{\wedge}, L^{\wedge}\right)_{X}$ and $K_{X}+(n-1) L$ is ample.

(0.9.2) Further assume that $\Gamma\left(\left(K_{X^{\wedge}}+(n-2) L^{\wedge}\right)^{t}\right)>0$ for some $t>0$. Then $K_{X}+(n-2) L$ is nef and there is a smooth surface $S$ which is the transverse intersection of $n-2$ general members of $|L|$ such that $S$ is a minimal model of nonnegative Kodaira dimension. Moreover the restriction $\left.\pi\right|_{S^{\wedge}}: S^{\wedge} \rightarrow S$ is the map into its minimal model.

(0.9.3) If $K_{X}+(n-2) L$ is nef and big, then $S$ is a surface of general type. Proof. (0.9.1) and (0.9.2) are proved in [So7, §4]. As to (0.9.3), note that $\left(K_{X}+(n-2) L\right)^{m}$ is spanned by global sections for $m \geq 5$ in view of $(0.8 .1)$. Let $\phi_{m}: X \rightarrow \mathbf{P}^{N}$ be the morphism associated to $\left|\left(K_{X}+(n-2) L\right)^{m}\right|$ and let $\phi_{m}^{\prime}$ be the restriction of $\phi_{m}$ to $S$. If $\operatorname{dim} \phi_{m}^{\prime}(S)<2$, then [So7, (0.3.2)] applies to say that $\operatorname{dim} \phi_{m}(X)=\operatorname{dim} \phi_{m}^{\prime}(S)<2$, which contradicts $K_{X}+(n-2) L$ to 
be nef and big. Then $\operatorname{dim} \phi_{m}^{\prime}(S)=2$, hence the $m$-canonical map of $S$ has a 2-dimensional image too.

(0.10) Castelnuovo's bound. Let $\left(X^{\wedge}, L^{\wedge}\right), S^{\wedge}, C^{\wedge}$ be as in (0.6). Assume that $\left|L^{\wedge}\right|$ embeds $X^{\wedge}$ in a projective space $\mathbf{P}^{N}, N \geq 4$, and let $d^{\wedge}=L^{\wedge n}$. Then $g\left(C^{\wedge}\right)=g\left(L^{\wedge}\right)$ and Castelnuovo's Lemma (see e.g. [Li-So, §0]) says that

$$
g\left(C^{\wedge}\right) \leq\left[\frac{d^{\wedge}-2}{N-n}\right]\left(d^{\wedge}-N+n-1-\left(\left[\frac{d^{\wedge}-2}{N-n}\right]-1\right) \frac{N-n}{2}\right)
$$

where $N=h^{0}\left(L^{\wedge}\right)-1=h^{0}\left(L_{S^{\wedge}}^{\wedge}\right)+n-3$ and $[x]$ means the greatest integer $\leq x$. We shall simply write Castel $\left(d^{\wedge}, h^{0}\left(L^{\wedge}\right)\right)$ to mean the right-hand side of (0.10.1).

Now, let $(X, L)$ be a reduction of $\left(X^{\wedge}, L^{\wedge}\right)$ and let $S=\pi\left(S^{\wedge}\right)$ as in (0.9). Look at the nonsingular curve $C=\pi\left(C^{\wedge}\right)$ and write $d=L^{n}$. Then the inequality

$$
g(C) \leq \sigma\left(d-h^{0}(L)+n-(\sigma-1) \frac{h^{0}(L)-1-n}{2}\right), \quad \sigma=\frac{d-2}{h^{0}(L)-1-n},
$$

holds true. Indeed, the base point set Bs $|L|$ of $|L|$ consists of a finite set of points, so that $|L|$ embeds $C$, out of $\operatorname{Bs}|L|$, as a curve of degree $d^{\prime} \leq d$ in some projective space. Thus

$$
g(C) \leq \operatorname{Castel}\left(d^{\prime}, h^{0}(L)\right) \leq \operatorname{Castel}\left(d, h^{0}(L)\right) .
$$

(0.11) Lemma. Let $\left(X^{\wedge}, L^{\wedge}\right), S^{\wedge}$ be as in (0.6). Assume that $\left|L^{\wedge}\right|$ embeds $X^{\wedge}$ in a projective space $\mathbf{P}^{N}$ and let $d^{\wedge}=L^{\wedge n}$. Then

$(0.11 .1)$ if $N=n+2, \quad d^{\wedge 2}-5 d^{\wedge}-10(g(L)-1)+12 \chi\left(\mathscr{O}_{S}\right)=2 K_{S^{\wedge}} \cdot K_{S^{\wedge}}$,

(0.11.2) if $N \geq n+3, \quad d^{\wedge 2}-5 d^{\wedge}-10(g(L)-1)+12 \chi\left(\mathscr{O}_{S}\right) \geq 2 K_{S^{\wedge}} \cdot K_{S^{\wedge}}$.

Proof. Let $N=n+2$. Then $h^{0}\left(L_{S^{\wedge}}^{\wedge}\right)=h^{0}\left(L^{\wedge}\right)-n+2$ and $\left|L_{S^{\wedge}}^{\wedge}\right|$ embeds $S^{\wedge}$ in $\mathbf{P}^{4}$, so $(0.11 .1)$ follows from the standard exact sequence of tangent bundles

$$
0 \rightarrow \mathscr{T}_{S^{\wedge}} \rightarrow i^{*} \mathscr{T}_{\mathbf{P}^{4}} \rightarrow \mathscr{N}_{S^{\wedge} / \mathbf{P}^{4}} \rightarrow 0,
$$

where $i: S \rightarrow \mathbf{P}^{4}$, as in [H, p. 434].

If $N \geq n+3$, then $\left|L_{S^{\wedge}}^{\wedge}\right|$ embeds $S^{\wedge}$ in a $\mathbf{P}^{N-n+2}=\mathbf{P}$. Look at the generic projection $S_{0}$ of $S^{\wedge}$ into a $\mathbf{P}^{4}$. The surface $S_{0}$ has a finite number \# of nodes as singular points. Let $S^{\prime}$ be the minimal desingularization of $S_{0}$. Then Lemma 2.2 of [Sol] applies to say that there exists a connected complex manifold $\mathscr{U}$ that contains $S^{\prime}$ as a submanifold and such that $S^{\prime} \rightarrow \mathbf{P}^{4}$ extends to a holomorphic immersion $\theta: \mathscr{U} \rightarrow \mathbf{P}^{4}$. Hence we have the exact sequence

$$
0 \rightarrow \mathscr{T}_{S^{\prime}} \rightarrow u^{*} \mathscr{T}_{\mathscr{U}} \rightarrow \mathscr{N}_{S^{\prime} / \mathscr{U}} \rightarrow 0
$$


where $\iota$ denotes the inclusion of $S^{\prime}$ in $\mathscr{U}$ and $\mathscr{T}_{\mathscr{U}}=\theta^{*} \mathscr{T}_{\mathbf{P}^{4}}$. Again the same argument as in [H, p. 434] works, noting that

$$
c_{2}\left(\mathscr{N}_{S^{\prime} / \mathscr{U}}\right)=\left(S^{\prime} \cdot S^{\prime}\right)_{\mathscr{U}}=\left(S^{\wedge} \cdot S^{\wedge}\right)_{\mathbf{P}}-2 \# .
$$

(0.12) Implications of the Barth-Lefschetz Theorem. Let $\left(X^{\wedge}, L^{\wedge}\right)$ be as in (0.6) and let $N=h^{0}\left(L^{\wedge}\right)-1$. Under the further assumption that $X^{\wedge}$ is a locally complete intersection a standard consequence of the Barth-Lefschetz Theorem gives the following conditions (see [Fl-La and So5]).

(0.12.1) $X^{\wedge}$ is simply connected if $2 n-N \geq 1$.

(0.12.2) If $2 n-N=1$, there exists an exact sequence

$$
0 \rightarrow \operatorname{Pic}\left(\mathbf{P}^{N}\right) \rightarrow \operatorname{Pic}(X) \rightarrow \mathscr{G} \rightarrow 0
$$

where $r: \operatorname{Pic}\left(\mathbf{P}^{N}\right) \rightarrow \operatorname{Pic}(X)$ is the restriction map and the cokernel $\mathscr{G}$ is a torsion free group.

$(0.12 .3)$ If $2 n-N \geq 2$, then $\operatorname{Pic}\left(\mathbf{P}^{N}\right) \cong \operatorname{Pic}(X)$ via $r$.

Let us recall the Riemann-Roch formula we use in case of isolated singularities.

(0.13) Riemann-Roch Theorem (see [F, Chapter 18.3]). Let $\left(X^{\wedge}, L^{\wedge}\right)$ be as in $(0.6)$ and let $(X, L)$ be a reduction of $\left(X^{\wedge}, L^{\wedge}\right)$. Assume $n=3$ and let $p: X^{\prime} \rightarrow X$ be a resolution of singularities. Since $X$ has a finite number of singular points, for a line bundle $\mathscr{L}$ on $X$, we have

$$
\chi(\mathscr{L})=\mathscr{L}^{3} / 6+p_{*} c_{1}^{\prime} \cdot \mathscr{L}^{2} / 4+p_{*}\left(c_{1}^{\prime 2}+c_{2}^{\prime}\right) \cdot \mathscr{L} / 12+\chi\left(\mathscr{O}_{X}\right)
$$

where $c_{i}^{\prime}=c_{i}\left(\mathscr{T}_{X^{\prime}}\right)$ are the Chern classes of $X^{\prime}, i=1,2$. Furthermore $\chi\left(\mathscr{O}_{X}\right)=$ $\chi\left(\mathscr{O}_{X^{\prime}}\right)+\sum n_{x}[x]$ with the sum over all singular points $x$ in $X$ and

$$
n_{x}=\ell\left(R^{1} p_{*} \mathscr{O}_{X^{\prime}}\right)_{x}-\ell\left(R^{2} p_{*} \mathscr{O}_{X^{\prime}}\right)_{x}
$$

where $\ell(\mathscr{F})_{x}$ denotes the length of the stalk of the sheaf $\mathscr{F}$ at $x$.

By using the projection formula one sees that

$$
p_{*} c_{1}^{\prime} \cdot \mathscr{L}^{2}=c_{1}^{\prime} \cdot\left(p^{*} \mathscr{L}\right)^{2}=-K_{X} \cdot \mathscr{L}^{2}
$$

and

$$
p_{*}\left(c_{1}^{\prime 2}\right) \cdot \mathscr{L}=c_{1}^{\prime 2} \cdot p^{*} \mathscr{L}=K_{X}^{2} \cdot \mathscr{L} \text {. }
$$

Therefore the Riemann-Roch formula takes its usual form

$$
\chi(\mathscr{L})=\mathscr{L}^{3} / 6-K_{X} \cdot \mathscr{L}^{2} / 4+\left(K_{X}^{2}+c_{2}(X)\right) \cdot \mathscr{L} / 12+\chi\left(\mathscr{O}_{X}\right)
$$

where $c_{2}(X)$ stands for $p_{*} c_{2}\left(X^{\prime}\right)$.

(0.14) Some special varieties. We say that a polarized pair $(X, \mathscr{L}), \mathscr{L}$ an ample line bundle on $X$, is a scroll over a variety $Y$ if there exists a surjective morphism $f: X \rightarrow Y$ such that $\left(F, \mathscr{L}_{F}\right) \cong\left(\mathbf{P}^{S}, \mathcal{O}(1)\right)$, with $s=n-\operatorname{dim} Y$, for every fibre $F$ of $f$. 
If $-K_{X}$ is ample we say that $X$ is a Fano variety. The largest positive integer $r$ such that $-K_{X} \approx r \mathscr{L}$, for some ample line bundle $\mathscr{L}$ on $X$, is the index of $X$. A polarized pair $(X, \mathscr{L})$ is said to be a Del Pezzo variety if $K_{X} \approx(1-n) \mathscr{L}$.

(0.15) The generalized Hodge index theorem. As a consequence of the usual Hodge index theorem we get the general results below which gives useful inequalities we need through the paper.

(0.15.1) Lemma. Let $X$ be an n-dimensional variety and let $M, N$ be line bundles on $X$, such that $M$ is ample and $N$ is nef. Then for $j=1, \ldots, n-1$

$$
\left(M^{j+1} \cdot N^{n-j-1}\right)\left(M^{j-1} \cdot N^{n-j+1}\right) \leq\left(M^{j} \cdot N^{n-j}\right)^{2} .
$$

Proof. First assume $N$ to be ample. Choose $D_{1}, \ldots, D_{j-1} \in|m M|, E_{1}, \ldots$, $E_{n-j-1} \in|m N|$. Then for $m \gg 0$ the transversal intersection of $D_{1}, \ldots, D_{j-1}$, $E_{1}, \ldots, E_{n-j-1}$ is a nonsingular surface $Y$. By using the usual Hodge index theorem one sees that

$$
\begin{aligned}
m^{2 n} & \left(M^{j+1} \cdot N^{n-j-1}\right)\left(M^{j-1} \cdot N^{n-j+1}\right) \\
& =m^{2 n}\left(M^{j-1} \cdot N^{n-j-1} \cdot M^{2}\right)\left(M^{j-1} \cdot N^{n-j-1} \cdot N^{2}\right) \\
& =\left(M_{Y} \cdot M_{Y}\right)\left(N_{Y} \cdot N_{Y}\right) \leq\left(M_{Y} \cdot N_{Y}\right)^{2} \\
& =m^{2 n}\left(M^{j-1} \cdot N^{n-j-1} \cdot M \cdot N\right) \\
& =m^{2 n}\left(M^{j} \cdot N^{n-j}\right)^{2}
\end{aligned}
$$

and hence we get the result.

Now assume $N$ to be nef. If $H$ is an ample line bundle on $X$, and $\varepsilon$ is a positive rational number, the Q-divisor $N+\varepsilon H$ is ample. Therefore, for $m \gg 0, m(N+\varepsilon H)$ is a very ample Cartier divisor. Then by the above

$$
\left(M^{j+1} \cdot(N+\varepsilon H)^{n-j-1}\right)\left(M^{j-1} \cdot(N+\varepsilon H)^{n-j+1}\right) \leq\left(M^{j} \cdot(N+\varepsilon H)^{n-j}\right)^{2}
$$

so we are done as soon as $\varepsilon$ goes to zero.

(0.15.2) Remark. If in (0.15.1) we have the equality and if $j=n-1$ also, then $N$ is numerically equivalent to a rational multiple of $M$. Indeed in this case we have

$$
\left(M_{Y} \cdot M_{Y}\right)\left(N_{Y} \cdot N_{Y}\right)=\left(M_{Y} \cdot N_{Y}\right)^{2}
$$

where $Y$ is the nonsingular surface obtained as the transversal intersection of $n-2$ general members of $|m M|, m \gg 0$. Let $a=N_{Y} \cdot N_{Y}$ and $b=M_{Y} \cdot N_{Y}$. Then $M_{Y} \cdot\left(a M_{Y}-b N_{Y}\right)=0$ and therefore by the usual Hodge index theorem one has $a M_{Y} \sim b N_{Y}$ since $M_{Y}$ is ample and $\left(a M_{Y}-b N_{Y}\right)^{2}=0$. It thus follows that $a M \sim b N$ by the first Lefschetz theorem on hyperplane sections.

Let $L_{1}, \ldots, L_{k}$ be nef line bundles on an $n$-dimensional variety $X$. Let $c_{1}(L)^{J}$ for a multi-index $J=\left(j_{1}, \ldots, j_{k}\right), j_{i} \geq 0, i=1, \ldots, k$, denote $\prod_{i=1}^{k} c_{1}\left(L_{i}\right)^{j_{i}}$. Let $|J|=\sum_{i=1}^{k} j_{i}$. 
(0.15.3) Theorem. With the notation as above, given a multi-index $J$ with $|J|=$ $n$ we have

$$
\begin{gathered}
c_{1}(L)^{J} \geq \prod_{i=1}^{k}\left[c_{1}\left(L_{i}\right)^{n}\right]^{j_{i} / n} \\
{\left[\left(\sum_{i=1}^{k} c_{1}\left(L_{i}\right)\right)^{n}\right]^{1 / n} \geq \sum_{i=1}^{k}\left[c_{1}\left(L_{i}\right)^{n}\right]^{1 / n} .}
\end{gathered}
$$

Proof. Relation (b) easily follows from (a). To prove (a), first note that by passing to a desingularization of $X$ we can assume that $X$ is smooth. Next note that it suffices to prove the theorem for ample line bundles. Indeed, let $H$ be an ample line bundle. If the results were true for ample line bundles, then for positive integers $m$

$$
\left(\prod_{i=1}^{k} c_{1}\left(m L_{i}+H\right)^{j_{i}}\right)^{n} \geq \prod_{i=1}^{k}\left(c_{1}\left(m L_{i}+H\right)^{n}\right)^{j_{i}} .
$$

Dividing by $m$ we get

$$
m^{n}\left(c_{1}(L)^{J}\right)^{n}+p(m) \geq m^{n} \prod_{i=1}^{k}\left(c_{1}\left(L_{i}\right)^{n}\right)^{j_{i}}+q(m)
$$

where $p(m), q(m)$ are degree $n-1$ polynomials in $m$ with Chern numbers of the $L_{i}$ and $H$ as coefficients. Dividing by $m$ and as soon as $m$ goes to the infinity, we find

$$
\left(c_{1}(L)^{J}\right)^{n} \geq \prod_{i=1}^{k}\left(c_{1}\left(L_{i}\right)^{n}\right)^{j_{i}}
$$

Further since the truth or falsity of the inequality above is uneffected by multiplying each $L_{i}$ by a large $m$ we can assume without loss of generality that all the $L_{i}$ are very ample.

Claim. If the theorem is true for $k=2$, then it is also true for $k>2$.

Proof of the Claim. We will do an induction on the number $t$ of very ample line bundles. Assume we know the result when $t \leq k-1$ where $k \geq 3$. Let $J=\left(j_{1}, \ldots, j_{k}\right)$. If $j_{k}=0$, then we are done. Otherwise choose $j_{k}$ general elements of $\left|L_{k}\right|$ and intersect them to get a $\left(n-j_{k}\right)$-dimensional submanifold $Y$ of $X$. Then by the induction hypothesis we have, for $t=k-1$,

$$
c_{1}(L)^{J}=\prod_{i=1}^{k-1} c_{1}\left(L_{i \mid Y}\right)^{j_{i}} \geq \prod_{i=1}^{k-1}\left[c_{1}\left(L_{i \mid Y}\right)^{n-j_{k}}\right]^{j_{i} /\left(n-j_{k}\right)}
$$

as well as, for $t=2$,

$$
\begin{aligned}
c_{1}\left(L_{i \mid Y}\right)^{n-j_{k}} & =c_{1}\left(L_{i}\right)^{n-j_{k}} \cdot c_{1}\left(L_{k}\right)^{j_{k}} \\
& \geq\left[c_{1}\left(L_{i}\right)^{n}\right]^{\left(n-j_{k}\right) / n}\left[c_{1}\left(L_{k}\right)^{n}\right]^{j_{k} / n} .
\end{aligned}
$$


Now the claim easily follows by combining $(*)$ with $(* *)$ and also recalling that $n-j_{k}=j_{1}+\cdots+j_{k-1}$.

Thus we are reduced to prove the statement in the case of very ample line bundles $L_{1}, L_{2}$ on $X$. Let $q_{j}=\log \left(c_{1}\left(L_{1}\right)^{j} \cdot c_{1}\left(L_{2}\right)^{n-j}\right)$. Then Lemma $(0.15 .1)$ above gives

$$
q_{j} \geq\left(q_{j-1}+q_{j+1}\right) / 2, \quad i=1, \ldots, n-1 .
$$

We have to prove

$$
q_{j} \geq(j / n) q_{n}+((n-j) / n) q_{0} .
$$

This follows immediately from (\#). Indeed let $f:[0, n] \rightarrow \mathbf{R}$ be the function such that $f(j)=q_{j}$ and which is extended by linearity between $i, i+1$ for $i=1, \ldots, n-1$. The relation (\#) simply says that the function $f(x)$ is convex. The desired relation (\#\#) is a consequence of convexity. To see this note that $(j / n) q_{n}+((n-j) / n) q_{0}$ is just the $y$ value of the point over $j$ of the line segment joining the points $\left(0, q_{0}\right)$ and $\left(n, q_{n}\right)$. So, by convexity, $f(j)$ is greater or equal to this value. This completes the proof.

For further background material we refer the reader to [So7, §0].

1. The $(n-2)$-PLURIDEgREES OF A POLARIZED PAIR

Throughout this section we fix a polarized pair $\left(X^{\wedge}, L^{\wedge}\right)$ as in (0.6). In [So7] a complete description of all cases where $K_{X^{\wedge}}+(n-1) L^{\wedge}$ is not nef and big is carried out. Thus we can assume $K_{X^{\wedge}}+(n-1) L^{\wedge}$ to be nef and big. Then there exists a reduction $(X, L)$ of $\left(X^{\wedge}, L^{\wedge}\right)$ in view of (0.9). From now on when $n \geq 3$ we can also assume $K_{X}+(n-2) L$ to be nef and big: indeed all cases where $n \geq 3$ and $K_{X}+(n-2) L$ are not nef and big are completely described again in [So7]. As in (0.8), write

$$
\mathscr{K}_{X}=K_{X}+(n-2) L
$$

and define the $(n-2)$-pluridegrees of $(X, L)$ (or simply pluridegrees, for short)

$$
d_{j}=\mathscr{K}_{X}^{j} \cdot L^{n-j}, \quad j=0, \ldots, n .
$$

For $j=0, d_{0}=d$ is the degree of $(X, L)$.

The fact that $\mathscr{K}_{X}$ is nef and big gives us some general inequalities. First note that

$$
d_{j}>0, \quad j=0, \ldots, n .
$$

(1.1) Lemma (The generalized Hodge index theorem). For $j=1, \ldots, n-1$ we have

$$
\begin{gathered}
d_{j}^{2} \geq d_{j+1} d_{j-1} ; \\
d_{j}^{2}=d_{j+1} d_{j-1} \quad \text { if } d_{1}^{2}=d d_{2} \\
d_{j}^{n} \geq d_{n}^{j} d^{n-j}
\end{gathered}
$$


Proof. (1.1.1) and (1.1.2) follow from (0.15.1) and (0.15.2) by taking $M=L$, $N=\mathscr{K}_{X}$, while (1.1.3) follows from $(0.15 .3)$ for $k=2, L_{1}=\mathscr{K}_{X}, L_{2}=L$, and $\left(j_{1}, j_{2}\right)=(j, n-j)$.

As a consequence we find a condition on $d_{2}$ and we also improve (0.8.1).

(1.2) Corollary. With the notation as above and $n \geq 3$ :

(1.2.1) The inequality $d_{2} \geq 3$ holds.

(1.2.2) $\mathscr{K}_{X}^{t}$ is spanned by global sections for $t \geq 3$.

Proof. Let $d^{\wedge}=L^{\wedge \cdot n}$ and note that $d \geq d^{\wedge}$. If $d_{2}=1$, then $d_{3}=d_{1}=$ $d=1$ by (1.1.1). Hence $d^{\wedge}=d=1$ which means that $\left(X^{\wedge}, L^{\wedge}\right)=(X, L) \cong$ $\left(\mathbf{P}^{n}, \mathscr{O}(1)\right)$, contradicting the nefness assumption on $\mathscr{K}_{X}$. Again by (1.1.1) we find for $d_{2}=2, d_{3}=4, d_{1}=d=1$ or $d_{3}=d_{1}=d=2$. The first case leads to the same contradiction as above. In the second case one has $d^{\wedge} \neq 1$ : otherwise $\left(X^{\wedge}, L^{\wedge}\right) \cong\left(\mathbf{P}^{n}, \mathscr{O}(1)\right)$ which contradicts the assumption that $K_{X^{\wedge}}+(n-1) L^{\wedge}$ is nef and big. Therefore $d=d^{\wedge}=2,\left(X^{\wedge}, L^{\wedge}\right)=(X, L) \cong\left(Q, \mathscr{O}_{Q}(1)\right), Q$ quadric hypersurface in $\mathbf{P}^{n+1}, \mathscr{K}_{Q}=\mathscr{O}_{Q}(-2)$, again a contradiction. This gives (1.2.1).

The statement in (1.2.2) is due to Reider's method. Indeed let $S^{\wedge}$ be as in (0.6). Then $S$ is a minimal model of a surface of general type (see (0.9.3)) and $K_{S}^{t}$ is spanned by global sections for $t \geq 3$ (see [Be]). Now the same argument works as in the proof of $(0.8 .1)$.

(1.3) Lemma. We have, for $j=1, \ldots, n$

$$
(n+2) d_{j} \geq(n-2) d_{j-1} .
$$

Proof. Since $\mathscr{K}_{X}$ is nef and big, $\chi\left(K_{X}+t \mathscr{K}_{X}\right)$ is a polynomial of degree $n$ and $\chi\left(K_{X}+t \mathscr{K}_{X}\right)=h^{0}\left(K_{X}+t \mathscr{K}_{X}\right)$ if $t$ is positive by $(0.1 .1)$. Hence there exists an integer $t, 1 \leq t \leq n+1$, such that $h^{0}\left(K_{X}+t \mathscr{K}_{X}\right)>0$. Writing $K_{X}+t \mathscr{K}_{X}=(t+1) \mathscr{K}_{X}-(n-2) L$ we find, for $j \geq 1$,

$$
(t+1) d_{j}-(n-2) d_{j-1}=\left((t+1) \mathscr{K}_{X}-(n-2) L\right) \cdot \mathscr{K}_{X}^{j-1} \cdot L^{n-j} \geq 0
$$

which gives the result.

As a consequence of (1.3) and genus formula (0.3.1) (see also (1.5) below) we easily get the bound

$$
d \leq(n+2)(g(L)-1) / n
$$

which is stronger than the bound $d<2 g(L)-2$ obtained from (0.3.1). Note also that if $X$ has nonnegative Kodaira dimension the stronger relation

$$
d \leq 2(g(L)-1) /(n-1)
$$

can be proved (see [Li-So, §0]). 
Let $A$ be the transversal intersection of $n-k-1$ general members of $|L|$. Then $A$ is a normal Gorenstein subscheme of $X$ of dimension $k+1$ and the adjunction formula gives

$$
\mathscr{K}_{X \mid A} \approx K_{A}+(k-1) L_{A}=\mathscr{K}_{A} .
$$

(1.4) Lemma. For $j=0, \ldots, n-1$ and $j$ even,

$$
d_{j}=d_{j+1} \bmod (2) \text {. }
$$

Proof. Let $A$ be as above. Then for $k=0, \ldots, n-1$ we have

$$
\left(K_{X}+k \mathscr{K}_{X}+(n-k-1) L\right) \cdot \mathscr{K}_{X}^{k} \cdot L^{n-k-1}=\left(K_{A}+k \mathscr{K}_{A}\right) \cdot \mathscr{K}_{A}^{k} .
$$

The left-hand member is equal to

$$
\left((k+1) \mathscr{K}_{X}+(1-k) L\right) \cdot \mathscr{K}_{X}^{k} \cdot L^{n-k-1}=(k+1) d_{k+1}-(k-1) d_{k} .
$$

By genus formula (0.3.1) it thus follows that

$$
(k+1) d_{k+1}-(k-1) d_{k}=2 g\left(\mathscr{K}_{A}\right)-2
$$

and $g\left(\mathscr{K}_{A}\right)$ is an integer by $(0.4)$. This gives the result.

Note that for $k=1, A$ can be assumed to be the smooth surface $S$ as in (0.9.2), (0.9.3), the minimal model of a surface of general type. It is easy to see that

First, the genus formula gives

$$
d=L_{S} \cdot L_{S} ; \quad d_{1}=K_{S} \cdot L_{S} ; \quad d_{2}=K_{S} \cdot K_{S} .
$$

$$
d_{1}=2 g(L)-2-d .
$$

From well-known properties of surfaces of general type (see [Ba-P-V, Chapter VII]) some further relations follow.

$$
d_{2}<9 \chi\left(\mathscr{O}_{S}\right) \text {. }
$$

Indeed Noether's formula $\chi\left(\mathscr{O}_{S}\right)=\left(c_{1}^{2}+c_{2}\right) / 12$ and the Miyaoka-Yau inequality $c_{1}^{2} \leq 3 c_{2}$ lead to $d_{2} \leq 9 \chi\left(\mathscr{O}_{S}\right)$. Note that the equality cannot happen. Otherwise $S$ is a ball quotient and hence standard arguments show that $S$ would be a $K(\pi, 1)$, thus contradicting [So3, 1.3].

Furthermore from Noether's inequality $p_{g}(S) \leq c_{1}^{2} / 2+2$ it follows that

$$
d_{2} \geq 2 \chi\left(\mathscr{O}_{S}\right)-6 \text {. }
$$

A result due to Beauville, the Beauville-Castelnuovo inequality, gives us conditions for $h^{0}\left(L_{S}\right) \geq \chi\left(L_{S}\right)$.

(1.8) Lemma. We have $h^{0}\left(L_{S}\right) \geq \chi\left(L_{S}\right)$ (respectively $h^{0}\left(L_{S^{\wedge}}^{\wedge}\right) \geq \chi\left(L_{S^{\wedge}}^{\wedge}\right)$ if one of the following holds:

(a) $d_{2}<3 \chi\left(\mathscr{O}_{S}\right)-10$;

(b) $\left(K_{S}-L_{S}\right) \cdot L_{S}<0$ (resp. $\left.\left(K_{S^{\wedge}}-L_{S^{\wedge}}^{\wedge}\right) \cdot L_{S^{\wedge}}^{\wedge}<0\right)$;

(c) $\left(K_{S}-L_{S}\right) \cdot\left(K_{S}+L_{S}\right)<0\left(\right.$ resp. $\left.\left(K_{S^{\wedge}}-L_{S^{\wedge}}^{\wedge}\right) \cdot\left(K_{S^{\wedge}}+L_{S^{\wedge}}^{\wedge}\right)<0\right)$. 
Proof. Clearly (b) or (c) leads to $h^{2}\left(L_{S}\right)=h^{0}\left(K_{S}-L_{S}\right)=0$ and hence $h^{0}\left(L_{S}\right) \geq$ $\chi\left(L_{S}\right)\left(\right.$ resp. $\left.h^{0}\left(L_{S^{\wedge}}^{\wedge}\right) \geq \chi\left(L_{S^{\wedge}}^{\wedge}\right)\right)$.

If $d_{2}<3 \chi\left(\mathscr{O}_{S}\right)-10$ then $d_{2}<3 p_{g}(S)-7$. Therefore Beauville's result mentioned above says in particular that the rational map associated to $\Gamma\left(K_{S}\right)$ is not birational. Since $L_{S}$ is very ample out off a finite set of points (resp. $L_{S^{\wedge}}^{\wedge}$ very ample) it thus follows that $h^{0}\left(K_{S}-L_{S}\right)=0\left(\right.$ resp. $\left.h^{0}\left(K_{S^{\wedge}}-L_{S^{\wedge}}^{\wedge}\right)=0\right)$ and we are done.

\section{THE EFFeCTIVITY OF $K_{X}+n \mathscr{K}_{X}$}

Throughout this section we fix a polarized pair $\left(X^{\wedge}, L^{\wedge}\right)$ of dimension $n \geq 3$ as in (0.6). Let $(X, L)$ be a reduction of $\left(X^{\wedge}, L^{\wedge}\right)$ and let $S^{\wedge}, S$ be as in (0.9). Then by the above $\mathscr{K}_{X}=K_{X}+(n-2) L$ can be assumed to be nef and big, so that $S$ is a smooth surface of general type by (0.9.3).

First, we need the following general fact. We have heard it attributed to Matsusaka. We give the proof in the three dimensional case: the general proof runs parallel.

(2.1) Lemma. Let $\mathscr{L}$ be an ample line bundle on a normal variety $Y$ of dimension $n$ such that $h^{0}(\mathscr{L}) \geq n$ and $\mathscr{L}^{n}=1$. Then $\mathscr{L}$ is spanned and $(Y, \mathscr{L}) \cong\left(\mathbf{P}^{n}, \mathscr{O}_{\mathbf{P}^{n}}(1)\right)$.

Proof $(n=3)$. Take a general element $D \in|\mathscr{L}|$ and let $C=\mathscr{L} \cdot \mathscr{L}$. Since $\mathscr{L}$ is ample and $\mathscr{L}^{3}=1$, the surface $D$ and the curve $C$ are both irreducible. From the exact sequences

$$
\begin{gathered}
0 \rightarrow \mathscr{O}_{X} \rightarrow \mathscr{L} \rightarrow \mathscr{L}_{D} \rightarrow 0, \\
0 \rightarrow \mathscr{O}_{D} \rightarrow \mathscr{L}_{D} \rightarrow \mathscr{L}_{C} \rightarrow 0
\end{gathered}
$$

we find $h^{0}\left(\mathscr{L}_{D}\right) \geq 3$ and $h^{0}\left(\mathscr{L}_{C}\right) \geq 2$. Since $\operatorname{deg} \mathscr{L}_{C}=\mathscr{L}^{3}=1$ and $C$ is irreducible it thus follows that $C \cong \mathbf{P}^{1}$. Therefore $\mathscr{L}_{C} \cong \mathscr{O}_{\mathrm{P}}(1)$ is spanned by global sections, so that $\mathscr{L}_{D}$ and $\mathscr{L}$ are also spanned. Furthermore $h^{0}\left(\mathscr{L}_{C}\right)=2$ implies that $h^{0}\left(\mathscr{L}_{D}\right)=3$, hence $h^{0}(\mathscr{L})=4$ and the morphism associated to $|\mathscr{L}|$ is an isomorphism.

As far as the effectivity of $K_{X}+n \mathscr{K}_{X}$ is concerned we have the following result. We thank the referee for suggesting an alternate proof to our original result for $n=3$. The special classes when the effectivity of $K_{X}+n \mathscr{K}_{X}$ fails are described in Proposition (2.3) below.

(2.2) Theorem. With the notation as above, $h^{0}\left(K_{X}+n \mathscr{K}_{X}\right)>0$ unless $n=3,4$ or 6 and there exists a proper modification $r: X \rightarrow \mathbf{P}^{n}$ such that $\mathscr{K}_{X}=r^{*} \mathscr{O}_{\mathbf{P}^{n}}(1)$. Proof. Since $\mathscr{K}_{X}$ is nef and big, $p(t)=\chi\left(t \mathscr{K}_{X}\right)$ is a polynomial of degree $n$. Note that $p(t)=h^{0}\left(t \mathscr{K}_{X}\right)$ for $t>0$ by (0.1.1) since

$$
t \mathscr{K}_{X}=K_{X}+(t-1) \mathscr{K}_{X}+(n-2) L
$$


where $(t-1) \mathscr{K}_{X}+(n-2) L$ is ample. Further $p(t)=(-1)^{n} h^{0}\left(K_{X}-t \mathscr{K}_{X}\right)$, for $t<0$, by the Serre duality and $(0.1 .1)$ combined with the fact that $\mathscr{K}_{X}$ is nef and big. Assume $p(-n)=(-1)^{n} h^{0}\left(K_{X}+n \mathscr{K}_{X}\right)=0$.

Note that either $p(t)$ or $p(t-n)$ is zero for $t=1, \ldots, n-1$. This follows because of the maps

$$
\Gamma\left(\mathscr{K}_{X}^{t}\right) \otimes \Gamma\left(K_{X} \otimes \mathscr{K}_{X}^{n-t}\right) \rightarrow \Gamma\left(K_{X} \otimes \mathscr{K}_{X}^{n}\right)
$$

and the latter group is zero by hypothesis. Thus since $p(-n)=0$ and since by the above remark the remaining zeros are between $-(n-1)$ and $(n-1)$ we conclude that $p(-(n-1))=0$. Indeed, if not, and if $(-1)^{n} p(-(n-1))>0$ then we would have a zero between $-n$ and $-n+1$. Therefore we must have $(-1)^{n} p(-(n-1))<0$, i.e. $h^{0}\left(K_{X}+(n-1) \mathscr{K}_{X}\right)<0$, a contradiction. This implies that $p(1) \neq 0$, since otherwise we would have more than $n$ zeros of $p(t)$ : i.e. we would have the zeros $-n,-(n-1)$ and for $t=1, \ldots, n-2$ one of $t$ or $t-n$. By looking at the maps

$$
\Gamma\left(\mathscr{K}_{X}^{t}\right) \otimes \Gamma\left(K_{X} \otimes \mathscr{K}_{X}^{n-t}\right) \rightarrow \Gamma\left(K_{X} \otimes \mathscr{K}_{X}^{n}\right)
$$

it thus follows that $p(t)=0$ for $t=-1, \ldots,-n$. Therefore

$$
p(t)=\lambda(t+1) \cdots(t+n) / n ! ; \quad \lambda=d_{n}=\mathscr{K}_{X}^{n} .
$$

Now by a lemma of Matsusaka [L-M, (2.1)],

$$
h^{0}\left(\mathscr{K}_{X}\right) \leq \operatorname{deg} \Phi(X)+\operatorname{dim} \Phi(X)
$$

where $\Phi: X \rightarrow \mathbf{P}_{\mathbf{C}}$ is the meromorphic map associated to $\Gamma\left(\mathscr{K}_{X}\right)$. Thus in our case $d_{n}(n+1)=p(1)=h^{0}\left(\mathscr{K}_{X}\right) \leq d_{n}+n$ which implies that

$$
1=d_{n}=\mathscr{K}_{X}^{n} ; \quad h^{0}\left(\mathscr{K}_{X}\right)=n+1 .
$$

By Corollary (0.8.3) there exists a proper modification $r: X \rightarrow Y$ such that $\mathscr{K}_{X}=r^{*} \mathscr{M}$ for some ample line bundle $\mathscr{M}$ on $Y$ satisfying $\mathscr{M}^{n}=1$ and $h^{0}(\mathscr{M})=n+1$ in view of (2.2.2). Hence Lemma (2.1) applies to give $(Y, \mathscr{M}) \cong$ $\left(\mathbf{P}^{n}, \mathscr{O}(1)\right)$.

Now, by comparing the coefficients of $t^{n-1}$ in (2.2.1) and in the RiemannRoch formula for $p(t)=\chi\left(t \mathscr{K}_{X}\right)$ we see that

$$
n(n+1) / 2 n !=-\mathscr{K}_{X}^{n-1} \cdot K_{X} / 2(n-1) !
$$

Since $-\mathscr{K}_{X}^{n-1} \cdot K_{X}=-d_{n}+(n-2) d_{n-1}$, we find $(n+2)=(n-2) d_{n-1}$ which leads to either $n=3, d_{n-1}=5 ; n=4, d_{n-1}=3$; or $n=6, d_{n-1}=2$. This proves the theorem.

(2.3) Proposition. With the relation as above let $h^{0}\left(K_{X}+n \mathscr{K}_{X}\right)=0$ and let $r: X \rightarrow \mathbf{P}^{n}$ be the proper modification with $n=3,4$ or 6 as in (2.2). Then $\chi\left(\mathscr{O}_{S}\right)=n+2$, where $S$ is the transversal intersection of $n-2$ general members 
of $|L|$, and the only possible values for the pluridegrees of $(X, L)$ are as in the table below.

\begin{tabular}{|c|c|c|c|c|c|c|c|}
\hline$n$ & $d$ & $d_{1}$ & $d_{2}$ & $d_{3}$ & $d_{4}$ & $d_{5}$ & $d_{6}$ \\
\hline 3 & $0<d \leq 125$ & $3 \leq d_{1} \leq 25$ & 5 & 1 & & & \\
\hline 4 & $0<d \leq 81$ & $3 \leq d_{1} \leq 27$ & 9 & 3 & 1 & & \\
\hline \multirow{2}{*}{6} & $0<d \leq 64$ & $4 \leq d_{1} \leq 32$ & 16 & & & & \\
\cline { 2 - 6 } & $0<d \leq 41$ & $4 \leq d_{1} \leq 24$ & 14 & & 4 & 2 & 1 \\
\hline
\end{tabular}

Furthermore $r$ is an isomorphism outside of a closed subset $B \subset \mathbf{P}^{n}$ such that $\operatorname{dim} B \leq 1$ if $n=3$ or 4 and $\operatorname{dim} B \leq 2$ if $n=6$.

Proof. For simplicity we give here the proof in the case $n=4$; indeed the case $n=6$ is an easy modification of it, while the case $n=3$ is straightforward.

Let $A$ be a general element of $|L|$. By looking over the proof of (2.2) we see that $p(-1)=h^{0}\left(2 K_{X}+2 L\right)=0$ and hence $h^{0}\left(K_{X}+L\right)=0$. Then the Kawamata-Viehweg vanishing theorem and the exact sequence

$$
0 \rightarrow K_{X} \otimes L \rightarrow K_{X} \otimes 2 L \rightarrow K_{A} \otimes L_{A} \rightarrow 0
$$

give us $\chi\left(K_{A} \otimes L_{A}\right)=h^{0}\left(K_{X} \otimes 2 L\right)=5$. Hence the exact sequence

$$
0 \rightarrow K_{A} \rightarrow K_{A} \otimes L_{A} \rightarrow K_{S} \rightarrow 0
$$

yields

$$
\chi\left(\mathscr{O}_{S}\right)=\chi\left(K_{S}\right)=5+\chi\left(\mathscr{O}_{A}\right) .
$$

Finally, from $p(0)=\chi\left(\mathscr{O}_{X}\right)=1$ and $\chi(-L)=\chi\left(K_{X}+L\right)=0$ we find $\chi\left(\mathscr{O}_{A}\right)=$ $\chi\left(\mathscr{O}_{X}\right)=1$, so $\chi\left(\mathscr{O}_{S}\right)=6$.

To prove the table, note that $d_{4}=1$ since $\mathscr{K}_{X}=r^{*} \mathscr{O}(1)$. Then $d_{3} \leq 3$ by (1.3). By combining (1.1) and (1.7) we find $d_{3}^{2} \geq d_{2} d_{4} \geq 6$ which gives $d_{3}=3$ and $d_{2} \leq 9$. Now we know that $\mathscr{K}_{X \mid S} \approx K_{S}$; further the restriction $r_{S}$ of $r$ to $S$ is the map associated to $\Gamma\left(K_{S}\right)$. To see this note that, since $X$ is birational to $\mathbf{P}^{4}$, then $h^{i}\left(\mathscr{O}_{X}\right)=0$ for $i>0$. Thus by the Lefschetz theorem $h^{i}\left(\mathscr{O}_{A}\right)=0$ for $0<i<3$ and hence $h^{1}\left(K_{X}+L\right)=h^{2}\left(\mathscr{O}_{A}\right)=h^{1}\left(K_{A}\right)=0$. Then $r_{S}$ is generically 1 to 1 so that $d_{2} \geq 3 \chi\left(\mathscr{O}_{S}\right)-10$ : otherwise it would be $d_{2}<3 p_{g}(S)-7$, this leading to a contradiction (see Lemma (1.8) and also [Ba-P-V, VII, (8.5)]). Hence $d_{2} \geq 8$ and therefore $d_{2}=9$ by parity Lemma (1.4). From (1.1) we find $3 \leq d_{1} \leq 27,0<d \leq 81$.

Let $B$ denote the closed subset of $\mathbf{P}^{4}$ where $r$ is not an isomorphism. Then $K_{X} \approx r^{*} K_{\mathrm{p}^{4}}+J$ for some effective line bundle $J$ such that $r(\operatorname{supp}(J))=B$ 
and $2 L \approx r^{*} \mathscr{O}(6)-J$. Note that the pluridegree $d_{2}$ of the pair $\left(\mathbf{P}^{4}, \mathscr{O}(1)\right)$ is $d_{2}\left(\mathbf{P}^{4}, \mathscr{O}(1)\right)=d_{2}=9$. We have

$$
\begin{aligned}
2 d_{2} & =L \cdot\left(r^{*} \mathscr{O}(6)-J\right) \cdot r^{*} \mathscr{O}(1) \cdot r^{*} \mathscr{O}(1) \\
& =r_{*} L \cdot \mathscr{O}(6) \cdot \mathscr{O}(1) \cdot \mathscr{O}(1)-r_{*} L \cdot B \cdot \mathscr{O}(1) \cdot \mathscr{O}(1) \\
& =2 d_{2}\left(\mathbf{P}^{4}, \mathscr{O}(1)\right)-b,
\end{aligned}
$$

where $b=B \cdot r_{*} L \cdot \mathscr{O}(1) \cdot \mathscr{O}(1)>0$ if $\operatorname{dim} B \geq 2$, a contradiction. This completes the proof.

The following examples show that numbers $d$ and $d_{1}$ can vary quite a lot. (2.4) Construction. Let $\mathscr{L}, \mathscr{M}$ be two spanned line bundles on an $n$-dimensional projective manifold $Y$. Assume $\mathscr{M}$ is very ample and $c_{1}(\mathscr{L})^{t} \neq 0$ as an element of $H^{2 t}(Y, \mathbf{Q})$. Then there exist $t$ elements $A_{1}, \ldots, A_{t}$ of $|\mathscr{L}|$ meeting transversally along a smooth connected codimension $t$ submanifold $B$ of $Y$. Let $p: X \rightarrow Y$ be the blowing-up of $Y$ along $B$, denote $E=p^{-1}(B)$, $\mathscr{L}^{\prime}=p^{*} \mathscr{L}-E$ and put $L=\mathscr{L}^{\prime} \otimes p^{*} \mathscr{M}$. Note that $L$ is very ample: indeed $\mathscr{L}^{\prime}$ is spanned by global sections and $\Gamma\left(\mathscr{L}^{\prime}\right)$ gives a map $q: X \rightarrow \mathbf{P}^{t-1}$ with connected fibres and with $\mathscr{L}^{\prime}=q^{*} \mathscr{O}_{\mathbf{P}^{t-1}}(1)$. Further $K_{X} \approx p^{*} K_{Y}+(t-1) E$. Thus $K_{X}+(t-1) L \approx p^{*}\left(K_{Y}+(t-1)(\mathscr{L}+\mathscr{M})\right)$. Now let $t=n-1$. Then

$$
\mathscr{K}_{X}=K_{X}+(n-2) L \approx p^{*}\left(K_{Y}+(n-2)(\mathscr{L}+\mathscr{M})\right) \text {. }
$$

(2.4.1) Example. Let $Y=\mathbf{P}^{3}, \mathscr{L}=\mathscr{O}_{\mathbf{P}^{3}}(4), \mathscr{M}=\mathscr{O}_{\mathbf{P}^{3}}(1)$ and let $F=$ $q^{*} \mathscr{O}_{\mathbf{P}^{1}}(1), \xi=p^{*} \mathscr{O}_{\mathbf{P}^{3}}(1)$. Then $L=F+\xi, \mathscr{K}_{X}=\xi$. Now $\xi^{3}=1, F^{3}=\xi \cdot F^{2}=$ $0, \xi^{2} \cdot F=4$, so we can compute $d=(F+\xi)^{3}=13 ; d_{1}=(F+\xi)^{2} \cdot \xi=9$; $d_{2}=(F+\xi) \cdot \xi^{2}=5 ; d_{3}=\xi^{3}=1$.

By taking $\mathscr{L}=\mathscr{O}_{\mathbf{p}^{3}}(1)$ and $\mathscr{M}=\mathscr{O}_{\mathbf{p}^{3}}(4)$ the same construction as above gives rise to a pair $(X, L)$ with pluridegrees $d_{3}=1, d_{2}=5, d_{1}=24$ and $d=112$.

(2.4.2) Example. Let $Y=\mathbf{P}^{4}, \mathscr{L}=\mathscr{O}_{\mathbf{P}^{4}}(2), \mathscr{M}=\mathscr{O}_{\mathbf{P}^{4}}(1)$ and let $F=$ $q^{*} \mathscr{O}_{\mathbf{P}^{2}}(1), \xi=p^{*} \mathscr{O}_{\mathbf{P}^{4}}(1)$. Then $L=F+\xi, \mathscr{K}_{X}=\xi$. Now $\xi^{4}=1, \xi^{3} \cdot F=2$, $\xi^{2} \cdot F^{2}=4, \xi \cdot F^{3}=F^{4}=0$, so we find $\left(d, d_{1}, d_{2}, d_{3}, d_{4}\right)=(33,19,9,3,1)$.

(2.4.3) Example. Let $Y=\mathbf{P}^{6}, \mathscr{L}=\mathscr{M}=\mathscr{O}_{\mathbf{P} 6}(1)$. Here the same computation as above gives us $\left(d, d_{1}, d_{2}, d_{3}, d_{4}, d_{5}, d_{6}\right)=(57,31,16,8,4,2,1)$.

It is worth noting that something more can be said under some extra assumptions. We carried out this in the three dimensional case only.

(2.5) Remark $(n=3)$. By looking over the proofs of (2.2) and (2.3) one sees that, for $n=3$, the general element $S \in|L|$ has the same Hodge numbers $h^{p, q}=h^{q}\left(\Omega_{S}^{p}\right)$ as a smooth 5 th degree surface in $\mathbf{P}^{3}$. 
(2.6) Remark $(n=3)$. Assume $X$ in $(2.2)$ to be smooth. Then the results of [So2] apply to describe the exceptional locus $E$ of the proper modification $r: X \rightarrow \mathbf{P}^{3}$. Precisely, $\mathbf{P}^{3}$ is obtained from $X$ by blowing down $\mathbf{P}^{1}$-bundles to smooth curves or by contracting to points certain divisors explicitly described as: $\mathbf{P}^{2}, \mathbf{F}_{0}, \mathbf{F}_{1}$ or $\mathbf{F}_{2} \cup \mathbf{P}^{2}$, where $\mathbf{F}_{e}$ is the Hirzebruch surface $\mathbf{P}\left(\mathscr{O}_{\mathbf{P}^{1}} \oplus \mathscr{O}_{\mathbf{P}^{1}}(e)\right), e=0,1,2$. Furthermore by slicing $E$ with a general member $S \in|L|$ one gets either smooth sections of $\mathbf{P}^{1}$-bundles or reduced, irreducible curves of type $A_{1}, A_{2}, A_{3}$ or $D_{4}$ (see [So2, (0.5.2), (1.5.2) and (1.8.2)]).

(2.7) Remark $(n=3)$. Assume $X$ has only rational singularities and let $\mathscr{K}_{X}$ be ample. Then $d_{3}>1$ unless either

- $(X, L) \cong\left(\mathbf{P}^{3}, \mathscr{O}_{\mathbf{P}^{3}}(5)\right)$;

- $X$ is a Fano variety of index $2, K_{X} \approx-2 M$ for an ample line bundle $M$ on $X$ such that $L \approx 3 M, M^{3}=1$, or

- $(X, L)$ is a scroll over an elliptic curve, $L_{F} \approx \mathscr{O}_{F}(4)$ for every fibre $F \cong \mathbf{P}^{2}$.

As to the proof, let $d_{3}=1$. We can assume $h^{0}\left(K_{X}+3 \mathscr{K}_{X}\right)>0$; otherwise by (2.2) one has $(X, L) \cong\left(\mathbf{P}^{3}, \mathscr{O}_{\mathbf{P}^{3}}(5)\right)$ since $\mathscr{K}_{X}$ is ample. Hence $d_{2}=1,2,3$ or 4 in view of (2.8) below. Cases $d_{2}=2,4$ are excluded by (1.4) while $d_{2}=1$ yields $d=1$ by (1.1.1). It thus easily follows that $\left(X^{\wedge}, L^{\wedge}\right) \cong(X, L) \cong$ $\left(\mathbf{P}^{3}, \mathscr{O}_{\mathbf{P}^{3}}(1)\right)$, contradicting $\mathscr{K}_{X}$ being nef. So we can assume $d_{2}=3$ and hence the genus formula for $\mathscr{K}_{X}$ gives $g\left(\mathscr{K}_{X}\right)=1$. Then, by [Fj, Corollary 2], $\left(X, \mathscr{K}_{X}\right)$ is either a Del Pezzo variety or a scroll over an elliptic curve. In the former case $3 K_{X} \approx-2 L$, so one easily sees that $X$ is a Fano variety of index $2, K_{X} \approx-2 M, M$ an ample line bundle on $X, L \approx 3 M$ and $M^{3}=1$ (an explicit example can be found in [I-Sh, Theorem 6, (V)]). In the latter case $\mathscr{K}_{X \mid F} \approx \mathscr{O}_{F}(1)$, so that $L_{F} \approx \mathscr{O}_{\mathbf{P}^{2}}(4)$ for every fibre $F \cong \mathbf{P}^{2}$ and we are done.

Theorem (2.2) has some nice consequences. First, it allows us to improve (1.3).

(2.8) Proposition. With the notation as above, we have

$$
(n+1) d_{j} \geq(n-2) d_{j-1}, \quad j \geq 1,
$$

or $K_{X}+n \mathscr{K}_{X}$ is not effective and $(X, L)$ is as in (2.3). Furthermore $(n+1) d_{1}>$ $(n-2) d_{0}$ if $X$ is not a quadric hypersurface.

Proof. If $(X, L)$ is not as in (2.3), then $K_{X}+n \mathscr{K}_{X}=(n+1) \mathscr{K}_{X}-(n-2) L$ is effective. Since $\mathscr{K}_{X}$ is nef and $L$ is ample, one has for $j \geq 1$,

$$
(n+1) d_{j}-(n-2) d_{j-1}=\left((n+1) \mathscr{K}_{X}-(n-2) L\right) \cdot \mathscr{K}_{X}^{j-1} \cdot L^{n-j} \geq 0 .
$$

Now suppose $(n+1) d_{1}=(n-2) d_{0}$. Then we have $\left((n+1) K_{S}-(n-2) L_{S}\right) \cdot L_{S}=$ 0 . The ampleness of $L_{S}$ and the effectivity of $(n+1) K_{S}-(n-2) L_{S}$ imply that $(n+1) K_{S} \approx(n-2) L_{S}$. Therefore $(n+1) \mathscr{K}_{X} \approx(n-2) L$ by the first Lefschetz 
theorem on hyperplane sections, so that $(n+1) K_{X} \approx-n(n-2) L$. This easily gives $K_{X} \approx-n M,(n-2) L \approx(n+1) M$ for some ample line bundle $M$ on $X$, which means that $X$ is a quadric in a $\mathbf{P}^{n+1}$ (see also [So7, (0.2)] and [Fa-So, $(0.6 .1)])$.

Note that $(2.8)$ gives the bound

$$
d \leq \frac{(n+1)(2 g(L)-2)}{2 n-1} .
$$

Let $\sigma\left(X^{\wedge}, L^{\wedge}\right)$ be the spectral value of $\left(X^{\wedge}, L^{\wedge}\right)$ as in $(0.5)$. If $\sigma\left(X^{\wedge}, L^{\wedge}\right)$ $\leq 3$, all the reductions $(X, L)$ have been characterized in [So7] in the $n$-dimensional case. As a consequence of $(2.2)$ all of the pairs $(X, L)$ with $\sigma\left(X^{\wedge}, L^{\wedge}\right)<$ $(4 n+1) /(n+1)$ can also be described.

(2.9) Corollary. With the notation as, above, either $\sigma\left(X^{\wedge}, L^{\wedge}\right) \geq(4 n+1) /(n+1)$ or $K_{X}+n \mathscr{K}_{X}$ is not effective and $(X, L)$ is as in (2.3).

Proof. Assume $(X, L)$ is not as in (2.3). Then $K_{X}+n \mathscr{K}_{X}=(n+1) K_{X}+$ $n(n-2) L$ is effective and writing

$$
(n+1) K_{X}+n(n-2) L=(n+1)\left(K_{X}+\left(n+1-\frac{4 n+1}{n+1}\right) L\right)
$$

one sees that $\sigma\left(X^{\wedge}, L^{\wedge}\right)=\sigma(X, L) \geq(4 n+1) /(n+1)$.

As far as the smooth three dimensional case is concerned, some bounds for $\chi\left(\mathscr{O}_{X}\right)$ and $e(X)$ can be given.

Let $J_{1}(L)$ be the first holomorphic jet bundle of $L$ on $X$. Note that all the usual inequalities that hold for Chern classes of spanned bundles (see e.g. [F1]), hold also for $J_{1}(L)$ even though $J_{1}(L)$ is not spanned at the finite set of points where $L$ is not very ample. To see this we need the following general fact.

(2.10) Lemma. Let $Y$ be a normal variety of dimension $n$ and let $E$ be a rank $r \geq n$ vector bundle on $Y$. Assume that the sequence

$$
X \times \Gamma(E) \rightarrow E \rightarrow \bigoplus \mathbf{C}_{x} \rightarrow 0,
$$

where $x \in F$ and ev: $X \times \Gamma(E) \rightarrow E$ is defined by ev $(x, s)=s(x) \in E_{x}$ for any $(x, s) \in X \times \Gamma(E)$, is exact, $F$ is a finite set and $\mathbf{C}_{x}$ is a skyscraper sheaf supported at $x$ with a single copy of $\mathbf{C}$ as fibre. Then

(2.10.1) $c_{i}(E) \cdot Y_{i} \geq 0$ for all effective $i$-cycles $Y_{i}$ on $Y$;

(2.10.2) $\left(c_{1}^{2}(E)-c_{2}(E)\right) \cdot Y_{2} \geq 0$ for all effective 2-cycles $Y_{2}$ on $Y$.

Proof. In view of condition (*), any $n-1$ general sections $s_{1}, \ldots, s_{n-1}$ of $E$ give linearly independent vectors of $E_{x}$ restricted to any $x \in F$. Thus, for $i>1, c_{1}(E)$ is dual to a cycle $\mathscr{C}$ that moves without base points; indeed $\mathscr{C}$ is defined as

$$
\mathscr{C}=\left\{z \in X: s_{1}(z), \ldots, s_{n-1}(z) \text { are not linearly independent in } E_{z}\right\} .
$$


For $i=1$, note that $\operatorname{det}(E)$ is spanned outside $F$ and so $(\operatorname{det}(E))^{m}$ is spanned by global sections for $m \gg 0$. This shows (2.9.1). For (2.9.2) note that

$$
\left(c_{1}^{2}(E)-c_{2}(E)\right) \cdot Y_{2}=c_{1}(\xi)^{r+1} \cdot p^{*} Y_{2}
$$

where $p: \mathbf{P}(E) \rightarrow X$ is the usual projection and $\xi$ is the tautological line bundle on $\mathbf{P}(E)$. The above condition $(*)$ translates into $\xi$ being spanned by global sections outside of a finite set of points, so $\xi^{m}$ is spanned for $m \gg 0$. This implies $c_{1}(\xi)^{t} \cdot Z_{t} \geq 0$ for all effective $t$-cycles $Z_{t}$ on $\mathbf{P}(E)$, in particular for $Z_{t}=p^{*} Y_{2}$.

In (2.11.4) below, the numbers $d_{j}^{\wedge}$ are the pluridegrees of $\left(X^{\wedge}, L^{\wedge}\right)$ we define at the end of this section.

(2.11) Proposition. Assume that $X^{\wedge}$ is smooth of dimension three and $\left|L^{\wedge}\right|$ embeds $X^{\wedge}$ in a $\mathbf{P}^{N}$. Let $(X, L)$ be a reduction of $\left(X^{\wedge}, L^{\wedge}\right)$. Then the following relations hold:

$$
\begin{aligned}
& \left(3 d_{2}+2 d_{1}-d+12 \chi\left(\mathscr{O}_{S}\right)\right) / 32 \geq \chi\left(\mathscr{O}_{X}\right) \\
& 2 d_{3}+7 d_{2}+12 d_{1}-3 d-18 \chi\left(\mathscr{O}_{S}\right)+48 \chi\left(\mathscr{O}_{X}\right) \geq 0 \\
& \quad e(X) \leq 24 \chi\left(\mathscr{O}_{S}\right)+2 g(L)-2-2 d_{2}
\end{aligned}
$$

$$
e\left(X^{\wedge}\right) \geq 48 \chi\left(\mathscr{O}_{X}\right)-84 \chi\left(\mathscr{O}_{S}\right)-d^{\wedge 2}+20 d^{\wedge}+17 d_{1}^{\wedge}+11 d_{2}^{\wedge}+d_{3}^{\wedge}
$$

with equality if $N \leq 6$.

Proof. By looking over the proof of (2.2), we can assume that $K_{X}+t \mathscr{K}_{X}$ is effective for some $t=1,2$ or 3 ; otherwise it would be $d_{3}=1$ and hence $\chi\left(\mathscr{\sigma}_{X}\right)=1$. We can also assume that $t=3$ since for $t=1,2$ we find stronger relations. Then Lemma (2.10) leads to

$$
\begin{gathered}
c_{2}\left(J_{1}(L)\right) \cdot\left(4 K_{X}+3 L\right) \geq 0, \\
\left(c_{1}\left(J_{1}(L)\right)^{2}-c_{2}\left(J_{1}(L)\right)\right) \cdot\left(4 K_{X}+3 L\right) \geq 0, \\
c_{3}\left(J_{1}(L)\right) \geq 0 .
\end{gathered}
$$

The vector bundle $J_{1}(L)$ fits in an exact sequence

$$
0 \rightarrow \mathscr{T}_{X}^{*} \otimes L \rightarrow J_{1}(L) \rightarrow L \rightarrow 0
$$

where $\mathscr{T}_{X}^{*}$ is the cotangent bundle to $X$. A standard computation shows that

$$
\begin{gathered}
c_{2}\left(J_{1}(L)\right)=c_{2}(X)+3 K_{X} \cdot L+6 L \cdot L, \\
c_{1}\left(J_{1}(L)\right)=K_{X}+4 L
\end{gathered}
$$

where $c_{2}(X)=c_{2}\left(\mathscr{T}_{X}^{*}\right)$. Recall that by the Riemann-Roch theorem $(0.13 .1)$ one has $c_{2}(X) \cdot L=12 \chi\left(\mathscr{O}_{S}\right)-d_{1}-d_{2}, S$ general member of $|L|$. Then by using the equalities above, it is purely a mechanical computation to show that 
(2.11.5) and (2.11.6) lead to (2.11.1) and (2.11.2) respectively, while (2.11.7) gives (2.11.3).

Finally (2.11.4) is nothing but formula (0.7.2) in [Li-So], rewritten in terms of the numbers $d_{j}^{\wedge}$, if $N \leq 6$. For $N \geq 7$ a similar argument as in the proof of $(0.11)$ works.

(2.12) Example. As a consequence of (2.11) we can compute the number of sections of $\mathscr{K}_{X}$ in some particular cases. E.g., suppose that there exists a smooth threefold $X$ in $\mathbf{P}^{5}$ with invariants $g(L)=12, d=9, d_{1}=13, d_{2}=d_{3}=17$, $\chi\left(\mathscr{O}_{X}\right) \leq 9$ (compare with Table 1). One has $h^{1}\left(\mathscr{O}_{X}\right)=h^{1}\left(\mathscr{O}_{S}\right)$ and $p_{g}(S)=8$

\section{TABLE 1}

\begin{tabular}{|c|c|c|}
\hline$d_{j}$ 's & $d_{j}^{\wedge}{ }^{\prime} s$ & Comments \\
\hline$>0$ & $d_{j}-(-1)^{j} \gamma, j \geq 0$ & \multirow{4}{*}{$\mathscr{K}_{K}$ nef and big } \\
\hline $\begin{array}{l}d_{j}^{2} \geq d_{j+1} d_{j-1}, n-1 \geq j \geq 1 \\
d_{j}^{n} \geq d_{n}^{j} d^{n-j}, n-1 \geq j \geq 1\end{array}$ & & \\
\hline \multicolumn{2}{|l|}{$d_{2} \geq 3 ; d_{j} \geq 2$ if $j \neq n$} & \\
\hline$(n+2) d_{j} \geq(n-2) d_{j-1}, j \geq 1$ & $\begin{array}{l}(n+2) d_{j}^{\wedge} \geq(n-2) d_{j-1}^{\wedge}, \\
j \geq 1, j \text { odd }\end{array}$ & \\
\hline$d_{j}=d_{j+1} \bmod (2), j$ even & $d_{j}^{\wedge}=d_{j+1}^{\wedge} \bmod (2), j$ even & \multirow{2}{*}{ genus formula } \\
\hline$d_{1}=2 g(L)-2-d$ & $d_{1}^{\wedge}=2 g(L)-2-d^{\wedge}$ & \\
\hline$d_{2}<9 \chi\left(\mathscr{O}_{S}\right)$ & $d_{2}^{\wedge}<9 \chi\left(\mathscr{O}_{S}\right)$ & \multirow{2}{*}{$\begin{array}{l}\text { Miyaoka's inequality } \\
\text { Noether's theorem }\end{array}$} \\
\hline$d_{2} \geq 2 \chi\left(\theta_{S}\right)-6$ & & \\
\hline$d_{2} \geq 3 \chi\left(\mathscr{O}_{S}\right)-10$ & & $\begin{array}{l}\text { if } \Gamma\left(K_{S}\right) \text { gives a generically } 1-1 \\
\text { map (Beauville-Castelnuovo } \\
\text { inequality) }\end{array}$ \\
\hline \multirow[t]{4}{*}{$(n+1) d_{j} \geq(n-2) d_{j-1}, j \geq 1$} & $\begin{array}{l}(n+1) d_{j}^{\wedge} \geq(n-2) d_{j-1}^{\wedge} \\
j \geq 1, j \text { odd }\end{array}$ & $\begin{array}{l}\text { unless } n=3,4 \text { or } 6 \text { and there } \\
\text { exists proper effective } \\
\text { modification } r: X \rightarrow \mathbf{P}^{n} \text { with } \\
\left.\mathscr{K}_{X}=r^{*} \mathscr{O}(1) \text { (see }(2.2),(2.3)\right) \\
(X \text { is not a quadric) }\end{array}$ \\
\hline & $\begin{array}{l}(n+1) d_{j}^{\wedge}-(n-2) d_{j-1}^{\wedge} \\
\geq-(-1)^{j}(2 n-1) \gamma, j \geq 1\end{array}$ & if $h^{0}\left(K_{X}+n \mathscr{K}_{X}\right)>0$ \\
\hline & $\begin{array}{l}2 d_{2}^{\wedge}=d^{\wedge 2}-5 d^{\wedge}- \\
10(g(L)-1)+12 \chi\left(\mathscr{O}_{S}\right)\end{array}$ & $X^{\wedge} \subset \mathbf{P}^{N}, N=n+2$ \\
\hline & $\begin{array}{l}2 d_{2}^{\wedge} \leq d^{\wedge 2}-5 d^{\wedge}- \\
10(g(L)-1)+12 \chi\left(\mathscr{O}_{S}\right)\end{array}$ & $X^{\wedge} \subset \mathbf{P}^{N}, N \geq n+3$ \\
\hline
\end{tabular}


for a general member $S \in|L|$. From (2.11.1) we find $h^{2}\left(\mathscr{O}_{X}\right) \leq 5$ so that the exact sequence

$$
0 \rightarrow L^{-1} \rightarrow \mathscr{O}_{X} \rightarrow \mathscr{O}_{S} \rightarrow 0
$$

gives $h^{0}\left(\mathscr{K}_{X}\right) \geq 3$.

(2.13) Remark. Assume $\left(X^{\wedge}, L^{\wedge}\right)=(X, L)$ and $X$ smooth of dimension three. By (2.11) we can conclude that cases $g(L)=10$ or 12 with $\chi\left(\mathscr{O}_{S}\right)=1$ and $g(L)=11$ with $\chi\left(\mathscr{\sigma}_{S}\right)=2$ do not occur if $X \subset \mathbf{P}^{N}, N \leq 6$. E.g. look at the case $g(L)=11, \chi\left(\mathscr{O}_{S}\right)=2$. From (2.11.1), (2.11.2) we find $-1 \leq \chi\left(\mathscr{O}_{X}\right) \leq 1$ and (2.10.3) gives $e(X) \leq 60$, contradicting (2.11.4).

Let us go back to the general case of dimension $n \geq 3$. Let $\pi: X^{\wedge} \rightarrow X$ be the blowing up of $X$ as in (0.7) and let $\gamma$ be the number of points of $X$ blown up. As done in $\S 1$ for the pair $(X, L)$, write

$$
\mathscr{K}_{X^{\wedge}}=K_{X^{\wedge}}+(n-2) L^{\wedge}
$$

and define as usual the pluridegrees of $\left(X^{\wedge}, L^{\wedge}\right)$ as

$$
d_{j}^{\wedge}=\mathscr{K}_{X^{\wedge}}^{j} \cdot L^{\wedge(n-j)}, \quad j=0, \ldots, n .
$$

Then one has

$$
d_{j}^{\wedge}=d_{j}-(-1)^{j} \gamma, \quad j=0, \ldots, n .
$$

All the properties of pluridegrees $d_{j}$ 's proved in $\S \S 1$ and 2 hold true for the $d_{j}^{\wedge}$ 's except those which need the nefness and bigness of $\mathscr{K}_{X}$. So numbers $d_{j}^{\wedge}$ 's may be negative and properties (1.1), (1.2), (1.3), (1.7), (2.8) and (2.11) do not work for the $d_{j}^{\wedge}$ 's. Let us only point out that (2.14) and (1.3) lead to

$$
(n+2) d_{j}^{\wedge}-(n-2) d_{j-1}^{\wedge} \geq(-1)^{j+1} 2 n \gamma, \quad j \geq 1,
$$

while, if $K_{X}+n \mathscr{K}_{X}$ is effective, then (2.14) and (2.8) easily yield

$$
(n+1) d_{j}^{\wedge}-(n-2) d_{j-1}^{\wedge} \geq(-1)^{j+1}(2 n-1) \gamma, \quad j \geq 1 .
$$

Table 1 summarizes all the relations in terms of the pluridegrees $d_{j}$ 's, $d_{j}^{\wedge}$ 's we have found except the bounds for $g(L), \chi\left(\mathscr{O}_{X}\right), e(X)$ and $e\left(X^{\wedge}\right)$ given in (0.10) and (2.11) respectively.

\section{NUMERICAL CLASSIFICATION OF SMOOTH THREEFOLDS}

Let $(X, L)$ be a reduction of $\left(X^{\wedge}, L^{\wedge}\right)$ of dimension $n=3$ and let $S^{\wedge}$, $C^{\wedge}, S, C$ be as in $\S 0$. Recall that $K_{X^{\wedge}}+2 L^{\wedge}$ and $\mathscr{K}_{X}=K_{X}+L$ are assumed to be nef and big. Further suppose that $X^{\wedge}$ is smooth and $\left|L^{\wedge}\right|$ embeds $X^{\wedge}$ in a projective space $\mathbf{P}^{N}, N \geq 5$. Our aim is to list all possible values for the numerical invariants introduced in the paper for low values of sectional genus $g(L)$.

Note that we need the smoothness assumption only to use (0.12) and (2.11). 
(3.1) Remark. Case $N=4$ can be easily carried out. Indeed $X^{\wedge}$ is embedded by $\left|L^{\wedge}\right|$ as a hypersurface of degree $d^{\wedge}$ in $\mathbf{P}^{4}$, so that

$$
K_{X^{\wedge}}+2 L^{\wedge} \approx \mathscr{O}_{X^{\wedge}}\left(d^{\wedge}-3\right) \text {. }
$$

Since $K_{X^{\wedge}}+2 L^{\wedge}$ is nef and big it has to be $d^{\wedge} \geq 4$. Let $S^{\wedge}$ be a general member of $\left|L^{\wedge}\right|$. Then $K_{S^{\wedge}} \approx \mathscr{K}_{X^{\wedge} \mid S^{\wedge}} \approx \mathscr{O}_{S^{\wedge}}\left(d^{\wedge}-4\right)$ and $d_{1}^{\wedge}=K_{S^{\wedge}} \cdot L_{S^{\wedge}}^{\wedge}=$ $d^{\wedge}\left(d^{\wedge}-4\right)$. The genus formula gives

$$
2 g(L)-2=d^{\wedge}+d^{\wedge}\left(d^{\wedge}-4\right)=d^{\wedge}\left(d^{\wedge}-3\right) .
$$

Thus the possibilities for $\left(d^{\wedge}, g(L)\right)$ are $\left(d^{\wedge},\left(d^{\wedge}\left(d^{\wedge}-3\right)+2\right) / 2\right)$. Note that case $d^{\wedge}=4, g(L)=3$ is excluded: otherwise the genus formula yields $d_{1}^{\wedge}=0$ and hence we get the contradiction $d_{1}<0$. Then $d^{\wedge} \geq 5$, so in particular $K_{X^{\wedge}} \approx \mathscr{O}_{X^{\wedge}}$ or is very ample. Note also that for $d^{\wedge}=5,6,7, \ldots$ the genera are $g(L)=6,10,15, \ldots$, respectively.

From Castelnuovo's bound ( 0.10$)$, by writing

$$
\left[\left(d^{\wedge}-2\right)(N-3)\right]=\frac{d^{\wedge}-2-\varepsilon}{N-3}, \quad 0 \leq \varepsilon \leq N-4,
$$

we infer that

$$
d^{\wedge} \geq \frac{N-1}{2}+\sqrt{\left(2(N-3) g(L)+\left(\frac{N-5}{2}-\varepsilon\right)^{2}\right)}, \quad 0 \leq \varepsilon \leq N-4,
$$

which leads to

$$
d^{\wedge} \geq \begin{cases}(N-1) / 2+\sqrt{(2(N-3) g(L)+1 / 4)} & \text { if } N-5 \text { is odd } \\ (N-1) / 2+\sqrt{2(N-3) g(L)} & \text { if } N-5 \text { is even } .\end{cases}
$$

The Hodge index theorem (1.1) gives us $d^{2 / 3} \leq d_{1}$, so that

$$
d+d^{2 / 3} \leq 2 g(L)-2
$$

by the genus formula. Therefore, since $d \geq d^{\wedge}$, we find from (3.2), (3.3)

$$
2+2 \sqrt{g(L)}+(2+2 \sqrt{g(L)})^{2 / 3} \leq 2 g(L)-2
$$

which implies that

$$
g(L) \geq 7 \text {. }
$$

(3.5) Claim. Cases $g(L)=7,8$ do not occur.

Proof. If $g(L)=7$, formulas (3.2), (3.3) and (1.5) give $d=d^{\wedge}=8$ and $d_{1}=4$. Then one has $d_{3}=1, d_{2}=2$ by (1.1.1). Again from (3.2) we see that $N=h^{0}\left(L_{S^{\wedge}}^{\wedge}\right)=5$, so $(0.11 .1)$ applies to give a contradiction.

If $g(L)=8,(3.2),(3.3)$ yield $8 \leq d^{\wedge} \leq d \leq 9$ and from (1.1.1) we easily rule out case $d=9$. Therefore $d=d^{\wedge}=8$ and the same argument as above leads to a contradiction. 
TABLE 2

\begin{tabular}{|c|c|c|c|c|c|c|c|c|}
\hline$g(L)$ & $d^{\wedge}$ & $d$ & $d_{1}$ & $d_{2}$ & $d_{3}$ & $\gamma$ & $\chi\left(\mathscr{\theta}_{S}\right)$ & H.i.r. \\
\hline \multirow{3}{*}{9} & 8 & 8 & 8 & 8 & 8 & 0 & 6 & 0 \\
\hline & 9 & \multirow{2}{*}{10} & \multirow{2}{*}{6} & \multirow{2}{*}{3} & \multirow{2}{*}{1} & 1 & 4 & \multirow{2}{*}{6} \\
\hline & 10 & & & & & 0 & 3 & \\
\hline \multirow{2}{*}{10} & 9 & 9 & 9 & 9 & 9 & \multirow{2}{*}{0} & 6 & 0 \\
\hline & 10 & 10 & 8 & 4 & 2 & & 4 & 24 \\
\hline \multirow{4}{*}{11} & 10 & 11 & 9 & 6 & 2 or 4 & 1 & 5 & 15 \\
\hline & \multirow{2}{*}{11} & 13 & 7 & 3 & 1 & 2 & 3 & 10 \\
\hline & & 11 & 9 & 7 & & 0 & 4 & 4 \\
\hline & 12 & 12 & 8 & 4 & 2 & 0 & 2 & 16 \\
\hline \multirow{6}{*}{12} & 9 & 9 & 13 & 17 & $5 \leq d_{3} \leq 21$, odd & 0 & 9 & 16 \\
\hline & & 10 & 12 & 6 & & 0 & 6 & 84 \\
\hline & 10 & 11 & 11 & 7 & 3 & 1 & 6 & 44 \\
\hline & & 10 & 12 & 12 & $4 \leq d_{3} \leq 12$, even & 0 & 7 & 24 \\
\hline & 11 & 11 & 11 & 8 & \multirow{2}{*}{2 or 4} & 0 & 5 & 33 \\
\hline & 12 & 13 & 9 & 6 & & 1 & 3 & 3 \\
\hline \multirow{11}{*}{13} & \multirow{2}{*}{10} & 11 & 13 & 14 & $4 \leq d_{3} \leq 14$, even & 1 & 8 & 15 \\
\hline & & 10 & 14 & 9 & $5 \leq d_{3} \leq 25$, odd & 0 & 9 & 6 \\
\hline & \multirow{4}{*}{11} & 14 & 10 & 6 & 2 & 3 & 5 & 16 \\
\hline & & 11 & 13 & 9 & 3 or 5 & 0 & 6 & 70 \\
\hline & & 12 & 12 & 10 & 4,6 , or 8 & 1 & 6 & 24 \\
\hline & & 11 & 13 & 15 & $5 \leq d_{3} \leq 17$, odd & 0 & 7 & 4 \\
\hline & \multirow{5}{*}{12} & 15 & 9 & 3 & 1 & 3 & 3 & 36 \\
\hline & & 16 & 8 & 4 & 2 & 4 & 3 & 0 \\
\hline & & 12 & 12 & 6 & 2 & 0 & 4 & 72 \\
\hline & & 13 & 11 & 7 & 3 & 1 & 4 & 30 \\
\hline & & 12 & 12 & 12 & 12 & 0 & 5 & 0 \\
\hline \multirow{13}{*}{14} & 10 & 10 & 16 & 20 & $6 \leq d_{3} \leq 24$, even & 0 & 10 & 56 \\
\hline & \multirow{5}{*}{11} & 13 & 13 & 6 & 2 & 2 & 6 & 91 \\
\hline & & 14 & 12 & 7 & 3 & 3 & 6 & 46 \\
\hline & & 11 & 15 & 10 & 4 or 6 & 0 & 7 & 115 \\
\hline & & 12 & 14 & 11 & 3,5, or 7 & 1 & 7 & 64 \\
\hline & & 11 & 15 & 16 & $4 \leq d_{3} \leq 16$, even & 0 & 8 & 49 \\
\hline & \multirow{4}{*}{12} & 12 & 14 & 7 & 3 & 0 & 5 & 112 \\
\hline & & 13 & 13 & 8 & 2 or 4 & 1 & 5 & 65 \\
\hline & & 14 & 12 & 9 & 3 or 5 & 2 & 5 & 18 \\
\hline & & 12 & 14 & 13 & $5 \leq d_{3} \leq 11$, odd & 0 & 6 & 40 \\
\hline & \multirow{3}{*}{13} & 14 & 12 & & & 1 & 3 & 60 \\
\hline & & 15 & 11 & 7 & 3 & 2 & 3 & 16 \\
\hline & & 13 & 13 & 11 & $3 \leq d_{3} \leq 9$, odd & 0 & 4 & 26 \\
\hline
\end{tabular}


By using all the above relations it is purely a mechanical procedure to check which invariants occur. We check this for threefolds $\left(X^{\wedge}, L^{\wedge}\right)$ in $\mathbf{P}^{N}$ in the following cases: $N=5$ and $g(L) \leq 14 ; N=6,7$ or 8 and $g(L) \leq 13$; $N=9$ and $g(L) \leq 14 ; N=10$ and $g(L) \leq 16$. We have carried these computations out using Oregon Pascal on a VAX 750 of the College of Science of the University of Notre Dame. We do not include here the program which is, however, available on request.

As an example, it should be worthwhile to list the invariants we obtain for threefolds in $\mathbf{P}^{5}$ of log-general type and with $h^{0}\left(K_{X}+3 \mathscr{K}_{X}\right)>0$. In Table 2 , "H.i.r." stands for the Hodge index relation $d_{1}^{2}=d d_{2}$. Note that whenever $d=d_{1}=d_{2}$, it also follows from (1.1.2) that $d_{3}=d_{j}$ for $j=0,1,2$.

Added in proof. Note that cases $\left(g(L), d^{\wedge}\right)=(13,10),(14,10)$ in the table of threefolds in $\mathbf{P}^{5}$ do not occur. Indeed, as pointed out to us by J. Rathmann, for codimension 2 manifolds $\left(X^{\wedge}, L^{\wedge}\right)$ in $\mathbf{P}^{N}$ the stronger bound $g(L) \leq$ $\frac{d^{\wedge}\left(d^{\wedge}-3\right)}{6}+1$ holds true whenever $g(L)$ does not reach the maximum with respect to the Castelnuovo bound (see L. Gruson and C. Peskine, Genre des courbes de l'espace projectif, Algebraic Geometry, Proceedings, Tromsö, Norway 1977, Lecture Notes in Math., vol. 687, Springer, 1978).

Furthermore the third author has shown in the paper On the nonemptyness of the adjoint linear system of a hyperplane section of a threefold, to appear in Crelle's J., that if $K_{X}+L$ is nef and big, then given a smooth surface $S \in|L|$, it follows that

$$
2 h^{0}\left(K_{X}+L\right)>\chi\left(\mathscr{\odot}_{S}\right)
$$

By using this and further arguments three more cases $\left(g(L), d^{\wedge}\right)=(9,9)$, $(9,10),(11,12)$ can be ruled out. Moreover explicit examples of three-folds in $\mathbf{P}^{5}$ can be constructed by using some of liaison's techniques. We refer for this to the preprint Threefolds of degree 9 and 10 in $\mathbf{P}^{5}$ by M. Beltrametti, $\mathbf{M}$. Schneider and A. J. Sommese.

\section{REFERENCES}

[Ba-P-V] W. Barth, C. Peters and A. Van de Ven, Compact complex surfaces, Ergeb. Math. Grenzgeb., vol. 4, Springer-Verlag, New York, 1984.

[Bo] E. Bombieri, Canonical models of surfaces of general type, Inst. Hautes Études Sci. Publ. Math. 42 (1973), 171-219.

[Be] A. Beauville, Letter on Reider's method, March 17, 1986.

[Fa-So] M. L. Fania and A. J. Sommese, On the minimality of hyperplane sections of Gorenstein threefolds, Proceedings of the 1984 Notre Dame Several Complex Variable Conference in honor of W. Stoll, Vieweg.

[Fj] T. Fujita, On polarized manifolds whose adjoint bundles are not semipositive, Algebraic Geometry, Sendai, 1985, Adv. Stud. in Pure Math., vol. 10, North-Holland, Amsterdam, 1987, pp. 167-178.

[Fl] W. Fulton, Intersection theory, Ergeb. Math. Grenzgeb., vol 2, Springer-Verlag, New York, 1984. 
[Fl-La] W. Fulton and R. Lazarsfeld, Connectivity and its applications in algebraic geometry, Proceedings, University of Illinois at Chicago Circle, 1980, Lecture Notes in Math., vol. 862, Springer-Verlag, 1981.

[H] R. Hartshorne, Algebraic geometry, Graduate Texts in Math., vol. 52, Springer-Verlag, New York, 1977.

[I-Sh] V. A. Iskovskih and V. V. Shokurov, Biregular theory of Fano 3-folds, Proceedings, Copenhagen 1978, Lecture Notes in Math., vol. 732, Springer-Verlag, New York, 1979.

[L-M] D. Lieberman and D. Mumford, Matsusaka's big theorem, Algebraic Geometry Conference, Arcata 1974, Proc. Sympos. Pure Math., vol. 29, Amer. Math. Soc., Providence, R.I., 1975, pp. 513-530.

[Li-So] E. L. Livorni and A. J. Sommese, Threefolds of non negative Kodaira dimension with sectional genus less than or equal to 15, Ann. Scuola Norm. Sup. Pisa Cl. Sci (4) 13 (1986), 537-557.

[R] L. Roth, On the projective classification of surfaces, Proc. London Math. Soc. (2) 42 (1937), $142-170$.

[So1] A. J. Sommese, Submanifolds of abelian varieties, Math. Ann. 233 (1978), 229-256.

[So2] _ Configurations of -2 rational curves on hyperplane sections of projective threefolds, Classification of Algebraic and Analytic Manifolds, Progress in Math., vol. 39, BirkhäuserVerlag, 1980, pp. 465-497.

[So3] _ _ Hyperplane sections, Proceedings of the Algebraic Geometry Conference, University of Illinois at Chicago Circle, 1980, Lecture Notes in Math., vol. 862, Springer-Verlag, 1981, pp. 232-271.

[So4] __, On the minimality of hyperplane sections of projective threefolds, J. Reine Angew. Math. 392 (1981), 16-41.

[So5] __ Complex subspaces of homogeneous complex manifolds-II: Homotopy results, Nagoya Math. J. 86 (1982), 101-129.

[So6] _ Ample divisors on normal Gorenstein surfaces, Abh. Math. Sem. Univ. Hamburg 55 (1985), 151-170.

[So7] __, On the adjunction theoretic structure of projective varieties, Complex Analysis and Algebraic Geometry, Proceedings, Göttingen 1985, Lecture Notes in Math., vol. 1194 SpringerVerlag, 1986, pp. 175-213. ITALY

Dipartimento di Matematica, Università di Genova, Via L. B. Alberti, 4-16132 Genova, ITALY

Dipartimento di Matematica, Universita de l'Aquila, Via Roma, 33-67100 L'Aquila,

Department of Mathematics, University of Notre Dame, Notre Dame, Indiana 46556 